\title{
FINITE TIME ANALYTICITY FOR THE TWO- AND THREE-DIMENSIONAL RAYLEIGH-TAYLOR INSTABILITY
}

BY

\author{
C. SULEM AND P. L. SULEM
}

\begin{abstract}
The Rayleigh-Taylor instability refers to the dynamics of the interface between two ideal irrotational fluids of different densities superposed one over the other and in relative motion. The well-posedness of this problem is considered for two- and three-dimensional flows in the entire space and in the presence of a horizontal bottom. In the entire space, finite time analyticity of the interface is proven when the initial interface has sufficiently small gradients and is flat at infinity. In the presence of a horizontal bottom, the initial interface corrugations has also to be small initially but it is not required to vanish at infinity.
\end{abstract}

1. Introduction. A fluid of constant density $\rho_{-}$and horizontal velocity $u_{-}$ superposed over a fluid of constant density $\rho_{+}$and velocity $u_{+}$parallel to $u_{-}$ corresponds to a stationary solution to the Euler equation. But this configuration is linearly unstable for any perturbation of the interface of wave number $\vec{k}$ in the direction of the flow with modulus $[\mathbf{1}]$

$$
|k|>\frac{4 \alpha}{1-\alpha^{2}} \frac{g}{\left(u_{+}-u_{-}\right)^{2}} .
$$

The coefficient $\alpha=\left(\rho_{+}-\rho_{-}\right) /\left(\rho_{+}+\rho_{-}\right)$is generally called the Atwood ratio; $g$ denotes the gravity field. When the two fluids have the same density $(\alpha=0)$, the problem is often referred to as the Kelvin-Helmholz instability. In this case, small corrugation of any wave number is amplified exponentially. As a consequence, the linear problem is well posed only for an analytic initial interface, and thus generally only during a finite time [2]. In contrast, for the water wave problem where the lower fluid is much denser than the upper one $(\alpha=1)$, the interface is linearly stable, suggesting that nonanalytic initial free surfaces may be considered.

Well-posedness for the nonlinear water waves has been investigated by many authors. Existence during a finite time has been established in spaces of analytic functions [3-6], and also in Sobolev spaces and Hölder spaces [7-9]. They either transform the fluid region on a simple domain by means of a conformal mapping (two-dimensional problem) or follow the evolution of the free surface in Lagrangian coordinates. The Rayleigh-Taylor problem $(\alpha \neq 1)$ in two-dimensions has been considered in $[\mathbf{1 0}]$ where a finite time analyticity result is presented. The proof, which in places is only sketched, uses scales of spaces of analytic functions à la Ovsjannikov [11]. The present paper also deals with the well-posedness of the Rayleigh-Taylor problem and extends to it an analysis previously done for the Kelvin-Helmholtz

Received by the editors November 8, 1983.

1980 Mathematics Subject Classification. Primary 76E30.

Key words and phrases. Raleigh-Taylor instability, analyticity, well-posedness. $0002-9947 / 85 \$ 1.00+\$ .25$ per page 
problem $[\mathbf{1 2}]$. Both two- and three-dimensional flows are considered and a class of analytic initial conditions larger than in $[\mathbf{1 0}]$ is permitted. In addition, periodicity is not required. In $\S \S 2$ and 3 , we derive for two- and three-dimensional flows, a close system of equations for the interface and the vorticity density. This is done by writing the momentum equation for inhomogeneous flow in the sense of distributions. When the densities of the two fluids are different $(\alpha \neq 0)$, the equation for the vorticity density is of Fredholm type. In $\S 4$, we adapt to this problem Nishida's formulation $[\mathbf{1 3}, \mathbf{1 4}]$ of an abstract Cauchy-Kowalevski theorem in scales of Banach spaces $[\mathbf{1 1}, \mathbf{1 5}]$. In $\S 5$, this theorem is used to prove analyticity during a finite time for the Rayleigh-Taylor problem in the entire space, under the condition that the interface gradients are sufficiently small initially. When the interface is not periodic, it is assumed to be flat at infinity. The effect of a horizontal bottom is considered in $\S 6$. In this case, the initial corrugation has also to be sufficiently small but is not required to vanish at infinity. This is due to a faster decay of the Green function of the Poisson equation at large distances. Finally, a few open problems are discussed in $\S 7$.

2. Equations of motion of the interface in two dimensions. We consider a fluid of density $\rho_{-}$superposed on a fluid of density $\rho_{+}$. The flow is irrotational in each of the domains where the fluid is homogeneous but a velocity discontinuity at the interface is permitted. The momentum equation reads:

$$
\begin{aligned}
& \text { (a) } \frac{\partial}{\partial t}(\rho u)+\sum_{i} \frac{\partial}{\partial x_{i}}\left(\rho u_{i} u\right)+\nabla p-\rho \vec{g}=0, \\
& \text { (b) } \operatorname{div} \boldsymbol{u}=0
\end{aligned}
$$

where $\vec{g}=(0,-g)$ is the gravity field.

The jump conditions across the interface are easily derived from the equation satisfied by $\operatorname{Curl}(\rho u)$ in the sense of distribution. We denote by $\varphi(\vec{r}, t)$ a vectorial test-function perpendicular to the plane of the flow. It will be sometimes identified with its unique component. We assume that $\varphi$ has a compact support which does not intersect the possible bottom. We have

$$
\begin{aligned}
& \text { (a) } \rho u \operatorname{Curl} \frac{\partial \varphi}{\partial t} d \vec{r} d t+\int \rho u_{i} u \operatorname{Curl} \frac{\partial \varphi}{\partial x_{i}} d \vec{r} d t-\vec{g} \int \rho \operatorname{Curl} \varphi d \vec{r} d t=0, \\
& \text { (b) } \int(u \cdot \nabla) \varphi d \vec{r}=0
\end{aligned}
$$

For simplicity, we shall consider in this section the case of a two-dimensional flow. The three-dimensional problem is considered in $\S 3$.

The interface is represented by the equation

$$
r=r(\lambda, t), \quad \lambda \in R
$$

or when cartesian coordinates are used

$$
x=x(\lambda, t), \quad y=y(\lambda, t) .
$$

$N=\left(-y_{\lambda}, x_{\lambda}\right)$ is a vector normal to the interface. $n$ and $\tau$ are normal and tangent unit vectors, respectively. The vorticity density $\Omega(\lambda, t)$ on the interface is a vector 
perpendicular to the plane of the flow defined as

$$
\int \psi(r) \operatorname{Curl} u(r, t) d r=\int \psi(r(\lambda, t)) \Omega(\lambda, t) d \lambda,
$$

where $\psi(r)$ is a scalar test function.

The velocity in a point exterior to the interface is given by the Biot-Savart formula

$$
u(r, t)=\int \nabla G\left(r, r\left(\lambda^{\prime}, t\right)\right) \wedge \Omega\left(\lambda^{\prime}, t\right) d \lambda^{\prime},
$$

where $G$ is the Green function of the Laplace operator in the domain. In the absence of boundaries and when the velocity vanishes at infinity,

$$
G\left(r, r^{\prime}\right)=-\frac{1}{2 \pi} \ln \sqrt{\left(x-x^{\prime}\right)^{2}+\left(y-y^{\prime}\right)^{2}} .
$$

in the presence of a horizontal bottom,

$$
G\left(r, r^{\prime}\right)=-\frac{1}{2 \pi} \ln \sqrt{\frac{\left(x-x^{\prime}\right)^{2}+\left(y-y^{\prime}\right)^{2}}{\left(x-x^{\prime}\right)^{2}+\left(y+y^{\prime}\right)^{2}}}
$$

If the interface is sufficiently smooth, the velocity $u$ has limits $u^{ \pm}(\lambda, t)$ when $r$ tends in an arbitrary way from one or the other side to a point $r(\lambda, t)$ of the interface. In addition,

$$
V(\lambda, t) \stackrel{\text { def }}{=} \frac{u^{+}+u^{-}}{2}(\lambda, t)=f \nabla G\left(r(\lambda, t), r\left(\lambda^{\prime}, t\right)\right) \wedge \Omega\left(\lambda^{\prime}, t\right) d \lambda^{\prime},
$$

where $\int$ indicates that the integral is taken in the sense of Cauchy principal value.

We rewrite each integral in (2.2) and (2.5) as the sums of integrals in each of the two subdomains separated by the interface, and apply the Green formula to the different terms. In each of these domains, the flow is homogeneous and irrotational and the Euler equation is satisfied in a classical sense. The only remaining contributions thus come from the interface. We denote by $[f]=f^{+}-f^{-}$the jump across the interface of a function defined on one and the other side. (2.2)(b) and (2.5) imply

$$
\begin{aligned}
& \text { (a) }[u] \cdot N=0, \\
& \text { (b) }[u] \wedge N=\Omega
\end{aligned}
$$

and $(2.2)(\mathrm{a})$ is rewritten in the form

$$
\begin{aligned}
\int([\rho u] & \wedge N) \cdot \frac{\partial \varphi}{\partial t} d t d \lambda+\int\left(\left[\rho u_{i} u\right] \wedge N\right) \cdot \frac{\partial \varphi}{\partial x_{i}} d \lambda d t \\
& +\int\left[\operatorname{Curl}\left(\rho u_{i} u\right)\right] \cdot N_{i} \varphi d \lambda d t-[\rho] \int(g \wedge N) \cdot \varphi d \lambda d t=0
\end{aligned}
$$

In the first term of (2.8), we make the replacement

$$
\frac{\partial \varphi}{\partial t}(r(\lambda, t), t)=\frac{d \varphi}{d t}(r(\lambda, t), t)-\left(\frac{\partial r}{\partial t} \cdot \tau\right) \frac{\partial \varphi}{\partial \tau}-\left(\frac{\partial r}{\partial t} \cdot n\right) \frac{\partial \varphi}{\partial n}
$$


where $\partial / \partial \tau=(1 /\|N\|) \partial / \partial \lambda$. We rewrite the second term of $(2.8)$ in the form

$$
\begin{aligned}
& \int\left(\left[\rho u_{i} u\right]\right.\wedge N) \cdot \frac{\partial \varphi}{\partial x_{i}} d \lambda d t=\int(V \cdot n)([\rho u] \wedge N) \cdot \frac{\partial \varphi}{\partial n} d \lambda d t \\
&+\int\left\{(V \cdot \tau)[\rho u] \wedge N+([u] \cdot \tau) \frac{(\rho u)^{+}+(\rho u)^{-}}{2} \wedge N\right\} \cdot \frac{\partial \varphi}{\partial \tau} d \lambda d t
\end{aligned}
$$

In the third term, we use

$$
\begin{aligned}
{\left[\operatorname{Curl}\left(\rho u_{i} u\right)\right] N_{i}=} & N_{i}\left[\nabla u_{i} \wedge \rho u\right] \\
= & \left(n \cdot \frac{\partial V}{\partial \lambda}\right)[\rho u] \cdot n+\left(\tau \cdot \frac{\partial V}{\partial \lambda}\right)[\rho u] \cdot \tau \\
& +\left[u_{\tau}\right] \frac{(\rho u)^{+}+(\rho u)^{-}}{2} \cdot n+\frac{\partial}{\partial \lambda}([u] \cdot \tau) \frac{(\rho u)^{+}+(\rho u)^{-}}{2} \cdot \tau .
\end{aligned}
$$

After integration by parts, (2.8) becomes

$$
\begin{aligned}
\int\left\{-\frac{\partial}{\partial t}([\rho u]\right. & \wedge N)+\frac{\partial}{\partial \lambda}\left(\left(\frac{\partial r}{\partial t}-V\right) \cdot \tau[\rho u] \wedge n\right) \\
& -[u] \cdot \tau \frac{\partial}{\partial \lambda}\left(\frac{(\rho u)^{+}+(\rho u)^{-}}{2} \cdot \tau\right)+\left(n \cdot \frac{\partial V}{\partial \lambda}\right)[\rho u] \cdot n \\
& \left.+\left(\tau \cdot \frac{\partial V}{\partial \lambda}\right)[\rho u] \cdot \tau+([u] \cdot \tau) \frac{(\rho u)^{+}+(\rho u)^{-}}{2} \cdot n+[\rho] g \wedge N\right\} \cdot \varphi \\
& +\left\{\left(V-\frac{\partial r}{\partial t}\right) \cdot n[\rho u] \wedge N\right\} \cdot \frac{\partial \varphi}{\partial n}=0
\end{aligned}
$$

where the vectors perpendicular to the plane of the flow are identified with their unique component.

(2.12) is satisfied for any test function $\varphi$; the coefficients of $\varphi$ and $\partial \varphi / \partial n$ in (2.12) thus vanish. Expressing $(\rho u)^{+} \pm(\rho u)^{-}$in terms of $V$ and $\Omega$ one finally obtains

PROPOSITION 2.1. If during a period of time, the interface between two twodimensional ideal fluids of different densities superposed one over the other remains a smooth curve $\Gamma(t)=\{r=r(\lambda, t), \lambda \in R\}$ with a vorticity density $\Omega(\lambda, t)$, we have

(a) $\left(r_{t}-V\right) \cdot n=0$,

$$
\text { (b) } \begin{gathered}
\frac{\partial}{\partial t}\left\{\Omega+\alpha\left(V \cdot r_{\lambda}\right)\right\}+\frac{\partial}{\partial \lambda}\left\{\frac{1}{\left|r_{\lambda}\right|^{2}}\left(\left(V-r_{t}\right) \cdot r_{\lambda}\right)\left(\frac{\Omega}{2}+\alpha\left(V \cdot r_{\lambda}\right)\right)\right\} \\
-\alpha \frac{\partial}{\partial \lambda}\left\{\frac{\Omega^{2}}{8\left|r_{\lambda}\right|^{2}}-\frac{|V|^{2}}{2}\right\}+\alpha g y_{\lambda}=0
\end{gathered}
$$

In (2.13) subscripts denote partial derivatives and $n$ is a unit vector normal to $\Gamma$. If no boundary is present and the fluids are at rest at infinity

$$
V(\lambda, t)=\frac{1}{2 \pi} \int \frac{r(\lambda, t)-r\left(\lambda^{\prime}, t\right)}{\left|r(\lambda, t)-r\left(\lambda^{\prime}, t\right)\right|^{2}} \wedge \Omega\left(\lambda^{\prime}, t\right) d \lambda^{\prime}
$$

In the presence of a horizontal bottom $(r=(x, y), \bar{r}=(x,-y))$

$$
V(\lambda, t)=\frac{1}{2 \pi} \int\left(\frac{r(\lambda, t)-r\left(\lambda^{\prime}, t\right)}{\left|r(\lambda, t)-r\left(\lambda^{\prime}, t\right)\right|^{2}}-\frac{r(\lambda, t)-\bar{r}\left(\lambda^{\prime}, t\right)}{\left|r(\lambda, t)-\bar{r}\left(\lambda^{\prime}, t\right)\right|^{2}}\right) \wedge \Omega\left(\lambda^{\prime}, t\right) d \lambda^{\prime}
$$


If $\rho^{-}$and $\rho^{+}$are the densities of the upper and lower fluids, respectively, $\alpha=$ $\left(\rho^{+}-\rho^{-}\right) /\left(\rho^{+}+\rho^{-}\right)$.

The system (2.13), (2.14) simplifies when the interface can be resolved in the form $y=y(x, t)$. It becomes

$$
\begin{aligned}
& \text { (a) } \begin{aligned}
& y_{t}-V_{2}+y_{x} V_{1}=0, \\
& \text { (b) } \frac{\partial}{\partial t}\left\{\frac{\Omega}{2}+\alpha\left(V_{1}+y_{x} V_{2}\right)\right\}+\frac{\partial}{\partial x}\{ V_{1}\left(\frac{\Omega}{2}+\alpha\left(V_{1}+y_{x} V_{2}\right)\right) \\
&\left.+\alpha\left(\frac{\Omega^{2}}{8\left(1+y_{x}^{2}\right)}-\frac{|V|^{2}}{2}+g y\right)\right\}=0 .
\end{aligned}
\end{aligned}
$$

In the entire plane

$$
\begin{aligned}
& V_{1}(x, t)=-\frac{1}{2 \pi} f \frac{y(x, t)-y\left(x^{\prime}, t\right)}{\left(x-x^{\prime}\right)^{2}+\left(y(x, t)-y\left(x^{\prime}, t\right)\right)^{2}} \Omega\left(x^{\prime}, t\right) d x^{\prime} \\
& V_{2}(x, t)=\frac{1}{2 \pi} f \frac{x-x^{\prime}}{\left(x-x^{\prime}\right)^{2}+\left(y(x, t)-y\left(x^{\prime}, t\right)\right)^{2}} \Omega\left(x^{\prime}, t\right) d x^{\prime}
\end{aligned}
$$

in the presence of a horizontal bottom,

$$
\begin{aligned}
& V_{1}(x, t)=-\frac{1}{2 \pi} \int\left(\frac{y(x, t)-y\left(x^{\prime}, t\right)}{\left(x-x^{\prime}\right)^{2}+\left(y(x, t)-y\left(x^{\prime}, t\right)\right)^{2}}\right. \\
& \left.-\frac{y(x, t)+y\left(x^{\prime}, t\right)}{\left(x-x^{\prime}\right)^{2}+\left(y(x, t)+y\left(x^{\prime}, t\right)\right)^{2}}\right) \Omega\left(x^{\prime}, t\right) d x^{\prime} \\
& V_{2}(x, t)=\frac{1}{2 \pi} \int\left(\frac{x-x^{\prime}}{\left(x-x^{\prime}\right)^{2}+\left(y(x, t)-y\left(x^{\prime}, t\right)\right)^{2}}\right. \\
& \left.-\frac{x-x^{\prime}}{\left(x-x^{\prime}\right)^{2}+\left(y(x, t)+y\left(x^{\prime}, t\right)\right)^{2}}\right) \Omega\left(x^{\prime}, t\right) d x^{\prime} .
\end{aligned}
$$

Computing the time derivative in (2.15)(b) and using (2.15)(a), one obtains

PROPOSITION 2.2. When the Rayleigh-Taylor problem is considered in $R^{2}$ and the interface can be resolved in the form $y=y(x, t)$, the equations of motion become

where

$$
\begin{aligned}
& \text { (a) } y_{t}-V_{2}+y_{x} V_{1}=0 \\
& \text { (b) } \frac{1}{2} \frac{\partial \Omega}{\partial t}-\alpha A\{y\} \frac{\partial \Omega}{\partial t}+\frac{\partial F}{\partial x}=0
\end{aligned}
$$

$$
A\{y\} \frac{\partial \Omega}{\partial t}(x)=\int \frac{y(x, t)-y\left(x^{\prime}, t\right)-\left(x-x^{\prime}\right) y_{x}(x, t)}{\left(x-x^{\prime}\right)^{2}+\left(y(x, t)-y\left(x^{\prime}, t\right)\right)^{2}} \frac{\partial \Omega}{\partial t}\left(x^{\prime}, t\right) d x^{\prime}
$$

and

$$
\begin{aligned}
F(x, t)= & \frac{1}{2} V_{1}(x, t) \Omega(x, t)+\alpha\left(\frac{|V(x, t)|^{2}}{2}+\frac{\Omega^{2}(x, t)}{8\left(1+y_{x}^{2}(x, t)\right)}\right) \\
& +\alpha \int \frac{\left(x-x^{\prime}\right) \Omega\left(x^{\prime}, t\right)\left(V_{2}\left(x^{\prime}, t\right)-y_{x}(x, t) V_{1}\left(x^{\prime}, t\right)\right)}{\left(x-x^{\prime}\right)^{2}+\left(y(x, t)-y\left(x^{\prime}, t\right)\right)^{2}} d x^{\prime}+\alpha g y=0 .
\end{aligned}
$$

$V_{1}$ and $V_{2}$ are defined in (2.17). 
When a horizontal bottom is present, the equation for the vorticity density reads

$$
\frac{\partial \Omega}{\partial t}+B\{y\} \frac{\partial \Omega}{\partial t}=S\{\Omega, y\}
$$

with

$$
\begin{aligned}
S\{\Omega, y\}= & -\frac{\partial}{\partial x}\left(V_{1} \frac{\Omega}{2}+\alpha \frac{|V|^{2}}{2}+\frac{\alpha}{8} \frac{\Omega^{2}}{1+y_{x}^{2}}+\alpha g y\right) \\
& +\frac{1}{2 \pi} \frac{\partial}{\partial x} \int\left(V_{2}\left(x^{\prime}\right)-y_{x}\left(x^{\prime}\right) V_{1}\left(x^{\prime}\right)\right)\left(\frac{x-x^{\prime}}{\left(x-x^{\prime}\right)^{2}+\left(y(x, t)-y\left(x^{\prime}, t\right)\right)^{2}}\right) \\
& -\left(\frac{x-x^{\prime}}{\left(x-x^{\prime}\right)^{2}+\left(y(x, t)+y\left(x^{\prime}, t\right)\right)^{2}}\right) \Omega\left(x^{\prime}, t\right) d x^{\prime}
\end{aligned}
$$

and

$$
\begin{aligned}
& B\{y\} \frac{\partial \Omega}{\partial t}=\frac{\alpha}{2 \pi} \int\left(\frac{y(x)+y\left(x^{\prime}\right)-y_{x}(x)\left(x-x^{\prime}\right)}{\left(x-x^{\prime}\right)^{2}+\left(y(x)+y\left(x^{\prime}\right)\right)^{2}}\right. \\
&\left.-\frac{y(x)-y\left(x^{\prime}\right)-y_{x}(x)\left(x-x^{\prime}\right)}{\left(x-x^{\prime}\right)^{2}+\left(y(x)-y\left(x^{\prime}\right)\right)^{2}}\right) \frac{\partial \Omega}{\partial t}\left(x^{\prime}\right) d x^{\prime}
\end{aligned}
$$

Let us assume that $y(x, t)$ fluctuates around some mean value $h$. To isolate the linear contribution in the right-hand side of $(2.24)$ we write

$$
y(x, t)=h+\eta(x, t) .
$$

(2.22) becomes

$$
\frac{\partial \Omega}{\partial t}+\int \frac{2 h}{\left(x-x^{\prime}\right)^{2}+4 h^{2}} \frac{\partial \Omega}{\partial t}\left(x^{\prime}\right) d x^{\prime}=-C_{h}\{\eta\} \frac{\partial \Omega}{\partial t}+S\{\Omega, y\}
$$

with

$$
C_{h}\{\eta\} \frac{\partial \Omega}{\partial t}=B\{y\} \frac{\partial \Omega}{\partial t}-\int \frac{2 h}{\left(x-x^{\prime}\right)^{2}+4 h^{2}} \frac{\partial \Omega}{\partial t}\left(x^{\prime}\right) d x^{\prime} .
$$

The linear operator acting on $\partial \Omega / \partial t$ in the left-hand side of $(2.26)$ is easily inverted by taking the Fourier transform of the equation. We finally obtain

PROPOSITION 2.3. When the Rayleigh-Taylor problem is considered in the halfplane $y>0$, and the interface can be resolved in the form $y=y(x, t) \equiv h+\eta(x, t)$, the equations of motion read

$$
\begin{aligned}
& \text { (a) } \eta_{t}-V_{2}+\eta_{x} V_{1}=0 \\
& \text { (b) } \frac{\partial \Omega}{\partial t}+A\{\eta\} \frac{\partial \Omega}{\partial t}=F\{\Omega, \eta\}
\end{aligned}
$$

with

$$
\begin{gathered}
A\{\eta\} \frac{\partial \Omega}{\partial t}=C_{h}\{\eta\} \frac{\partial \Omega}{\partial t}+\int \mathcal{G}\left(x-x^{\prime}\right)\left(C_{h}\{\eta\} \frac{\partial \Omega}{\partial t}\left(x^{\prime}\right)\right) d x^{\prime}, \\
F\{\Omega, \eta\}=S\{\Omega, y\}+\int \mathcal{G}\left(x-x^{\prime}\right) S\{\Omega, y\}\left(x^{\prime}\right) d x^{\prime}
\end{gathered}
$$


The kernel $\mathcal{G}$ is given by

$$
\mathcal{G}(x)=\int_{-\infty}^{+\infty} e^{-i k x} \frac{\alpha e^{-2 h|k|}}{1+\alpha e^{-2 h|k|}} d k
$$

and thus decay like $|x|^{-2}$ when $x \rightarrow \pm \infty . V_{1}$ and $V_{2}$ are defined in (2.17) with $y=h+\eta ; C_{h}$ and $S$ are given in (2.27) and (2.22).

3. Equations of motion of the interface in three dimensions. The interface is represented by the equation $\left\{\vec{r}=r(\vec{\lambda}, t), \vec{\lambda}=\left(\lambda_{1}, \lambda_{2}\right) \in R^{2}\right\}$. The vorticity density $\Omega(\vec{\lambda}, t)$ is defined by

$$
\int \varphi(\vec{r}) \operatorname{Curl} u(\vec{r}, t) d \vec{r}=f \varphi(r(\vec{\lambda}, t)) \Omega(\vec{\lambda}, t) d \vec{\lambda},
$$

where $\varphi$ is a test function. Like in dimension 2 , when the interface is sufficiently smooth, we have

$$
V\{\vec{\lambda}, t\}=\frac{u^{+}+u^{-}}{2}(\lambda, t)=-\int \nabla G\left(\vec{r}(\vec{\lambda}, t), \vec{r}\left(\vec{\lambda}^{\prime}, t\right)\right) \wedge \Omega\left(\vec{\lambda}^{\prime}, t\right) d \vec{\lambda}^{\prime},
$$

where $u^{ \pm}$are the limits of the velocity $u(\vec{r}, t)$ when $\vec{r}$ tends from one or the other side to a point $\vec{r}(\vec{\lambda}, t)$ of the interface and $G$ is the Green function of the Laplace operator. When the problem is considered in $R^{3}$,

$$
G\left(\vec{r}, \vec{r}^{\prime}\right)=\frac{1}{4 \pi} \frac{1}{\left|\vec{r}-\vec{r}^{\prime}\right|} .
$$

When it is considered in half a space,

$$
G\left(\vec{r}, \vec{r}^{\prime}\right)=\frac{1}{4 \pi}\left(\frac{1}{\left|\vec{r}-\vec{r}^{\prime}\right|}-\frac{1}{\left|\vec{r}-\vec{r}^{\prime}\right|}\right)
$$

with $\vec{r}=\left(x_{1}, x_{2}, z\right)$ and $\overrightarrow{\vec{r}}=\left(x_{1}, x_{2},-z\right)$. a normal vector $N$ to the interface is $N=\partial r / \partial \lambda_{1} \wedge \partial r / \partial \lambda_{2}$. Using a Green' formula, (2.2) is rewritten as

$$
\begin{aligned}
& \int([\rho u] \wedge N) \cdot \frac{\partial \varphi}{\partial t} d \vec{\lambda} d t+\int\left(\left[\rho u_{i} u\right] \wedge N\right) \cdot \frac{\partial \varphi}{\partial x_{i}} d \vec{\lambda} d t \\
& \quad+\int\left[\operatorname{Curl}\left(\rho u_{i} u\right)\right] \cdot\left(N_{i} \varphi\right) d \vec{\lambda} d t+[\rho] \int(g \wedge N) \cdot \varphi d \vec{\lambda} d t=0 .
\end{aligned}
$$

Proceeding as in $[\mathbf{1 0}]$, we obtain the following system of equations:

(a) $\left(r_{t}-V\right) \cdot N=0$,

(b) $\quad-\frac{\partial}{\partial t}([\rho u] \wedge N)$

$$
\begin{aligned}
& +\sum_{j=1}^{2}(-1)^{j+1} \frac{\partial}{\partial \lambda_{j}}\left\{\frac{[\rho u] \wedge N}{\|N\|^{2}}\left(\frac{\partial r}{\partial t}, \frac{\partial r}{\partial \lambda_{\tilde{j}}}, N\right)-\frac{1}{\|N\|^{2}}\left(\frac{\partial r}{\partial \lambda_{\tilde{j}}} \wedge N\right)_{i}\left[\rho u_{i} u\right]\right\} \\
& +\left[\nabla u_{i} \wedge \rho u\right] N_{i}+\alpha \vec{g} \wedge N=0 .
\end{aligned}
$$


In $(3.6)$ (b), the subscript $\tilde{j}$ is 2 if $j=1$ and 1 if $j=2 ;(a, b, c)$ denotes the triple scalar product $a \cdot(b \wedge c)$. Furthermore,

$$
\begin{aligned}
\frac{1}{\|N\|^{2}}\left(\frac{\partial r}{\partial \lambda_{j}} \wedge N\right)_{i}\left[\rho u_{i} u\right] \wedge N= & \frac{1}{\|N\|^{2}}\left(V, \frac{\partial r}{\partial \lambda_{j}}, N\right)[\rho u] \wedge N \\
& -\left([u] \wedge \frac{\partial r}{\partial \lambda_{j}}\right) \wedge \frac{(\rho u)^{+}+(\rho u)^{-}}{2}
\end{aligned}
$$

and $[\mathbf{1 0}]$

$$
\begin{aligned}
{\left[\nabla u_{i} \wedge \rho u\right]=} & \|N\|\left(\left[\frac{\partial u}{\partial n}\right] \wedge \frac{(\partial u)^{+}+(\rho u)^{-}}{2}+\frac{\partial V}{\partial n} \wedge[\rho u]\right) \\
=\sum_{j=1}^{2}(-1)^{j+1}\left\{\frac{\partial}{\partial \lambda_{j}}\left(\frac{\partial r}{\partial \lambda_{\tilde{j}}} \wedge[u]\right) \wedge\right. & \frac{(\rho u)^{+}+(\rho u)^{-}}{2} \\
& \left.+\left(\frac{\partial r}{\partial \lambda_{\tilde{j}}} \wedge \frac{\partial V}{\partial \lambda_{j}}\right) \wedge[\rho u]\right\}
\end{aligned}
$$

We also express $(\rho u)^{+} \pm(\rho u)^{-}$in terms of $[u]$ and $V$, and notice that

$$
[u] \cdot \frac{\partial r}{\partial \lambda_{j}}=\frac{1}{\|N\|}\left(\frac{\partial r}{\partial \lambda_{j}}, N, \Omega\right)
$$

We thus obtain

PROPOSITION 3.1. If during a period of time, the interface between two ideal fluids of different densities superposed one over the other remains a smooth surface $S(t)=\left\{\vec{r}=r(\vec{\lambda}, t)\right.$ with $\left.\vec{\lambda}=\left(\lambda_{1}, \lambda_{2}\right) \in R^{2}\right\}$ with a smooth (vectorial) vorticity density $\Omega(\vec{\lambda}, t)$, one has

$$
\text { (a) } \begin{aligned}
&\left(V-\frac{\partial r}{\partial t}\right) \cdot N=0 \\
& \text { (b) } \frac{\partial}{\partial t}\left(\frac{\Omega}{2}+\alpha(V \wedge N)\right) \\
&+\sum_{j=1}^{2}(-1)^{j+1} \frac{\partial}{\partial \lambda}\left\{\frac{1}{\|N\|^{2}}\left(V-\frac{\partial r}{\partial t}, \frac{\partial r}{\partial \lambda_{j}}, N\right)\left(\frac{\Omega}{2}+\alpha V \wedge N\right)\right\} \\
&+(-1)^{j+1}\left\{\frac{1}{2\|N\|^{2}}\left(\frac{\partial r}{\partial \lambda_{j}}, N, \Omega\right)+\alpha\left(V \cdot \frac{\partial r}{\partial \lambda_{j}}\right)\right\} \frac{\partial V}{\partial \lambda_{j}} \\
&+\alpha(-1)^{j+1} \frac{\partial}{\partial \lambda_{j}}\left(\frac{V^{2}}{2}-\frac{[u]^{2}}{8}\right) \frac{\partial r}{\partial \lambda_{j}}-\alpha \vec{g} \wedge N=0
\end{aligned}
$$

where the jump of the velocity through the interface is given by

$$
[u]=\frac{1}{\|N\|^{2}} \sum_{j=1}^{2}(-1)^{j+1}\left(\Omega \cdot \frac{\partial r}{\partial \lambda_{j}}\right) \frac{\partial r}{\partial \lambda_{j}},
$$

and $V$ defined in (3.2). 
This system simplifies when the interface can be resolved in the form $z=z(\vec{x}, t)$ with $\vec{x}=\left(x_{1}, x_{2}\right)$. (3.10) becomes

$$
\begin{aligned}
& \text { (a) } \frac{\partial z}{\partial t}-V_{3}+\frac{\partial z}{\partial x_{1}} V_{1}+\frac{\partial z}{\partial x_{2}} V_{2}=0 \\
& \text { (b) } \frac{\partial}{\partial t}\left(\frac{\Omega}{2}+\alpha V \wedge N\right)=S
\end{aligned}
$$

with

$$
\begin{aligned}
-S= & \sum_{j=1}^{2} \frac{\partial}{\partial x_{j}}\left\{V_{j}\left(\frac{\Omega}{2}+\alpha V \wedge N\right)\right\}-\left\{\frac{\Omega_{j}}{2}+\alpha\left(V \cdot \frac{\partial r}{\partial x_{j}}\right)\right\} \frac{\partial V}{\partial x_{j}} \\
& +\alpha \frac{\partial}{\partial x_{j}}\left\{\frac{|V|^{2}}{2}-\frac{[u]^{2}}{8}\right\} \frac{\partial r}{\partial x_{j}}+\alpha g \wedge N .
\end{aligned}
$$

Let us first consider the problem in $R^{3}$. Developing $\partial(V \wedge N) / \partial t,(3.12)(\mathrm{b})$ is rewritten in the form

$$
\frac{\partial \Omega_{i}}{\partial t}+\alpha \sum_{j=1}^{3} B_{i j}\{z\} \frac{\partial \Omega_{j}}{\partial t}=E_{i}\{\Omega, z\} \quad(i=1,2,3) .
$$

The right-hand side of (3.14) is given by

$$
E=S+I+J
$$

with, for $i=1,2$,

$$
\begin{aligned}
I_{j}= & -\frac{\alpha}{4 \pi}(-1)^{j+1} \frac{\partial}{\partial x_{\tilde{j}}} \int \frac{\partial z(\vec{x}) / \partial t-\partial z\left(\vec{x}^{\prime}\right) / \partial t}{\left|r(\vec{x})-r\left(\vec{x}^{\prime}\right)\right|^{3}} \\
& \times\left\{\left(x_{2}-x_{2}^{\prime}\right) \Omega_{1}\left(\vec{x}^{\prime}\right)-\Omega_{2}\left(\vec{x}^{\prime}\right)\left(x_{1}-x_{1}^{\prime}\right)\right\} d \vec{x}^{\prime}, \\
I_{3}= & -\frac{\alpha}{4 \pi} \sum_{j=1}^{2} \frac{\partial}{\partial x_{j}} \int \frac{\partial z(\vec{x}) / \partial t-\partial z\left(\vec{x}^{\prime}\right) / \partial t}{\left|r(\vec{x})-r\left(\vec{x}^{\prime}\right)\right|^{3}} \\
& \times\left\{\left(x_{j}-x_{j}^{\prime}\right) \Omega_{3}\left(\vec{x}^{\prime}\right)-\Omega_{j}\left(\vec{x}^{\prime}\right)\left(z(\vec{x})-z\left(\vec{x}^{\prime}\right)\right)\right\} d \vec{x}^{\prime}
\end{aligned}
$$

and

$$
\begin{aligned}
J= & -\frac{\alpha}{4 \pi} \int \frac{\partial z(\vec{x}) / \partial t-\partial z\left(\vec{x}^{\prime}\right) / \partial t}{\left|r(\vec{x})-r\left(\vec{x}^{\prime}\right)\right|^{3}} \\
& \times\left\{3 \frac{r(\vec{x})-r\left(\vec{x}^{\prime}\right)}{\left|r(\vec{x})-r\left(\vec{x}^{\prime}\right)\right|^{2}}\left[\Omega\left(\vec{x}^{\prime}\right) \cdot\left(r(\vec{x})-r\left(\vec{x}^{\prime}\right)\right)\right]-\Omega\left(\vec{x}^{\prime}\right)\right\} d \vec{x}^{\prime} .
\end{aligned}
$$

In the left-hand side of (3.14),

$$
\begin{aligned}
B\{z\} \frac{\partial \Omega}{\partial t}=-\frac{1}{4 \pi} \int\{ & \left(N(\vec{x}) \cdot \frac{r(\vec{x})-r\left(\vec{x}^{\prime}\right)}{\left|r(\vec{x})-r\left(\vec{x}^{\prime}\right)\right|^{3}}\right) \frac{\partial \Omega}{\partial t}\left(\vec{x}^{\prime}\right) \\
& \left.-\frac{r(\vec{x})-r\left(\vec{x}^{\prime}\right)}{\left|r(\vec{x})-r\left(\vec{x}^{\prime}\right)\right|^{3}}\left(N(\vec{x}) \cdot \frac{\partial \Omega}{\partial t}\left(\vec{x}^{\prime}\right)\right)\right\} d x^{\prime} .
\end{aligned}
$$

For a given $z, B$ can be viewed as a linear integral operator acting on $\partial \Omega / \partial t$. Let $B_{i j}$ be the matricial elements of this operator. The kernels of $B_{3 j}(j=1,2,3)$ 
will be "small" of the interface gradients are "small". The kernels of $B_{13}$ and $B_{23}$ which operate on $\partial \Omega / \partial t$ have contributions which are independent of $\nabla z$. In these terms, we make the replacement

$$
\frac{\partial \Omega_{3}}{\partial t}=-\alpha \sum_{j=1}^{3} B_{3 j} \frac{\partial \Omega_{j}}{\partial t}+E_{3}\{\Omega, z\} .
$$

The resulting operator acting on $\partial \Omega_{j} / \partial t$ is then "small" with $\nabla z$.

PROPOSITION 3.2. If, during a period of time, the interface between two ideal fluids of different densities superposed one over the other is a smooth surface $z=$ $z(\vec{x}, t)$ with a smooth vorticity density $\Omega(\vec{x}, t)$, one has

$$
\begin{aligned}
& \text { (a) } \frac{\partial z}{\partial t}-V_{3}+\frac{\partial z}{\partial x_{1}} V_{1}+\frac{\partial z}{\partial x_{2}} V_{2}=0 \\
& \text { (b) } \frac{\partial \Omega_{j}}{\partial t}+\alpha A_{j}\{z\} \frac{\partial \Omega}{\partial t}=F\{\Omega, z\} \quad(j=1,2,3)
\end{aligned}
$$

with (no summation)

$$
F_{j}\{\Omega, z\}=E_{j}\{\Omega, z\}+\left(1-\delta_{3, j}\right) \int \frac{x_{j}-x_{j}^{\prime}}{\left|r(\vec{x})-r\left(\vec{x}^{\prime}\right)\right|^{3}} E_{3}\{\Omega, z\}\left(\vec{x}^{\prime}\right) d \vec{x}^{\prime}
$$

for $j=1,2$,

(a) $A_{j}\{z\} \frac{\partial \Omega}{\partial t}=-\frac{1}{4 \pi} \int \frac{1}{\left|r(\vec{x})-r\left(\vec{x}^{\prime}\right)\right|^{3}}$

$$
\begin{aligned}
& \times\left\{-\frac{\partial z}{\partial x_{j}}(\vec{x})\left(\left(x_{\tilde{j}}-x_{\tilde{j}}^{\prime}\right) \frac{\partial \Omega_{j}}{\partial t}\left(\vec{x}^{\prime}\right)-\left(x_{j}-x_{j}^{\prime}\right) \frac{\partial \Omega_{\tilde{j}}}{\partial t}\left(\vec{x}^{\prime}\right)\right)\right. \\
& \left.\quad+\left(z(\vec{x})-z\left(\vec{x}^{\prime}\right)\right) \frac{\partial \Omega_{j}}{\partial t}\left(\vec{x}^{\prime}\right)+\left(x_{j}-x_{j}\right) B_{3}\{z\} \frac{\partial \Omega}{\partial t}\left(\vec{x}^{\prime}\right)\right\} d \vec{x}^{\prime}
\end{aligned}
$$

(b) $A_{3}\{z\} \partial \Omega / \partial t=B_{3}\{z\} \partial \Omega / \partial t$

and

$$
V(\vec{x})=-\frac{1}{4 \pi} \int \frac{r(\vec{x})-r\left(\vec{x}^{\prime}\right)}{\left|r(\vec{x})-r\left(\vec{x}^{\prime}\right)\right|^{3}} \wedge \Omega\left(\vec{x}^{\prime}\right) d x^{\prime} .
$$

For simplicity, the temporal argument has been omitted.

When a horizontal bottom is present at $z=0$

$$
V(\vec{x})=-\frac{1}{4 \pi} \int_{R^{2}}\left(\frac{r(x)-r\left(x^{\prime}\right)}{\left|r(\vec{x})-r\left(\vec{x}^{\prime}\right)\right|^{3}}-\frac{r(x)-r\left(x^{\prime}\right)}{\left|r(\vec{x})-\bar{r}\left(x^{\prime}\right)\right|^{3}}\right) \wedge \Omega\left(\vec{x}^{\prime}\right) d \vec{x}^{\prime},
$$

where $\bar{r}(\vec{x})=\left(x_{1}, x_{2},-z\left(x_{1}, x_{2}\right)\right)$. When $\partial(V \wedge N) / \partial t$ is developed to write (3.12)(b) as a Fredholm equation, we get

$$
\partial \Omega / \partial t+\alpha B\{z\} \partial \Omega / \partial t=E\{\Omega, z\}
$$

where

$$
\begin{aligned}
B\{z\} \frac{\partial \Omega}{\partial t}=-\frac{1}{4 \pi} \int\{ & N(\vec{x}) \cdot\left(\frac{r(\vec{x})-r\left(\vec{x}^{\prime}\right)}{\left|r(\vec{x})-r\left(\vec{x}^{\prime}\right)\right|^{3}}-\frac{r(\vec{x})-\bar{r}\left(\vec{x}^{\prime}\right)}{\left|r(\vec{x})-\bar{r}\left(\vec{x}^{\prime}\right)\right|^{3}}\right) \frac{\partial \Omega}{\partial t}\left(\vec{x}^{\prime}\right) \\
& \left.-\left(N(\vec{x}) \cdot \frac{\partial \Omega}{\partial t}\left(\vec{x}^{\prime}\right)\right)\left(\frac{r(\vec{x})-r\left(\vec{x}^{\prime}\right)}{\left|r(\vec{x})-r\left(\vec{x}^{\prime}\right)\right|^{3}}-\frac{r(\vec{x})-\bar{r}\left(\vec{x}^{\prime}\right)}{\left|r(\vec{x})-\bar{r}\left(\vec{x}^{\prime}\right)\right|^{3}}\right)\right\} d \vec{x}^{\prime}
\end{aligned}
$$


and

$$
E=S+I+J
$$

with

$$
\begin{aligned}
-S=\sum_{j=1}^{2}\left\{V _ { j } \left(\frac{\Omega}{2}+\alpha V\right.\right. & \wedge N)+\left(\frac{\Omega_{j}}{2}+\alpha\left(V \cdot \frac{\partial r}{\partial x_{j}}\right)\right) \\
& \left.\times \frac{\partial V}{\partial x_{j}}-\alpha \frac{\partial}{\partial x_{j}}\left(\frac{|V|^{2}}{2}-\frac{\mid u]^{2}}{8}\right)\right\} \frac{\partial r}{\partial x_{j}}+\alpha g \wedge N .
\end{aligned}
$$

For $j=1,2$

$$
\begin{gathered}
I_{j}=\frac{-\alpha}{4 \pi}(-1)^{j+1} \frac{\partial}{\partial x_{j}} \int\left(\frac{\partial z(\vec{x}) / \partial t-\partial z\left(\vec{x}^{\prime}\right) / \partial t}{\left|r(\vec{x})-r\left(\vec{x}^{\prime}\right)\right|^{3}}-\frac{\partial z(\vec{x}) / \partial t+\partial z\left(\vec{x}^{\prime}\right) / \partial t}{\left|r(\vec{x})-\bar{r}\left(\vec{x}^{\prime}\right)\right|^{3}}\right) \\
\times\left(\left(x_{2}-x_{2}^{\prime}\right) \Omega_{1}\left(\vec{x}^{\prime}\right)-\Omega_{2}\left(\vec{x}^{\prime}\right)\left(x_{1}-x_{1}^{\prime}\right)\right) d \vec{x}^{\prime}
\end{gathered}
$$

and

$$
\begin{aligned}
I_{3}=-\frac{\alpha}{4 \pi} \sum_{j=1}^{2} \frac{\partial}{\partial x_{j}} \int\{ & \left(\frac{\partial z(\vec{x}) / \partial t-\partial z\left(\vec{x}^{\prime}\right) / \partial t}{\left|r(\vec{x})-r\left(\vec{x}^{\prime}\right)\right|^{3}}-\frac{\partial z(\vec{x}) / \partial t+\partial z\left(\vec{x}^{\prime}\right) / \partial t}{\left|r(\vec{x})-\bar{r}\left(\vec{x}^{\prime}\right)\right|^{3}}\right) \\
& \times\left(x_{j}-x_{j}^{\prime}\right) \Omega_{3}\left(\vec{x}^{\prime}\right)\left(x_{j}-x_{j}^{\prime}\right) \Omega_{3}\left(\vec{x}^{\prime}\right) \\
& -\left(\frac{\left(\partial z(\vec{x}) / \partial t-\partial z\left(\vec{x}^{\prime}\right) / \partial t\right)\left(z(\vec{x})-z\left(\vec{x}^{\prime}\right)\right)}{\left|r(\vec{x})-r\left(\vec{x}^{\prime}\right)\right|^{3}}\right. \\
& \left.\left.-\frac{\left(\partial z(\vec{x}) / \partial t+\partial z\left(\vec{x}^{\prime}\right) / \partial t\right)\left(z(\vec{x})+z\left(\vec{x}^{\prime}\right)\right)}{\left|r(\vec{x})-\bar{r}\left(\vec{x}^{\prime}\right)\right|^{3}}\right) \Omega_{j}\left(\vec{x}^{\prime}\right)\right\} d \vec{x}^{\prime},
\end{aligned}
$$

$$
\begin{aligned}
J= & -\frac{\alpha}{4 \pi} \int \frac{\partial z(\vec{x}) / \partial t-\partial z\left(\vec{x}^{\prime}\right) / \partial t}{\left|r(\vec{x})-r\left(\vec{x}^{\prime}\right)\right|^{3}} \\
& \times\left\{3 \frac{r(\vec{x})-r\left(\vec{x}^{\prime}\right)}{\left|r(\vec{x})-r\left(\vec{x}^{\prime}\right)\right|^{2}}\left(\Omega\left(\vec{x}^{\prime}\right) \cdot\left(r(\vec{x})-r\left(\vec{x}^{\prime}\right)\right)-\Omega\left(\vec{x}^{\prime}\right)\right)\right\} \\
& -\frac{\partial z(\vec{x}) / \partial t+\partial z\left(\vec{x}^{\prime}\right) / \partial t}{\left|r(\vec{x})-\bar{r}\left(\vec{x}^{\prime}\right)\right|^{3}}\left\{3 \frac{r(\vec{x})-\bar{r}\left(\vec{x}^{\prime}\right)}{\left|r(\vec{x})-r\left(\vec{x}^{\prime}\right)\right|^{2}}\left(\Omega\left(\vec{x}^{\prime}\right) \cdot\left(r(\vec{x})-\bar{r}\left(\vec{x}^{\prime}\right)\right)-\Omega\left(\vec{x}^{\prime}\right)\right)\right\} .
\end{aligned}
$$

Assume that $z(\vec{x}, t)$ fluctuates around some mean value $h$. As in two dimensions, we isolate the linear contribution by writing

$$
z(\vec{x}, t)=h+\eta(\vec{x}, t) .
$$

For the two first components of $B\{z\} \partial \Omega / \partial t$, we write

$$
\frac{z(\vec{x})+z\left(\vec{x}^{\prime}\right)}{\left|r(\vec{x})-\bar{r}\left(\vec{x}^{\prime}\right)\right|^{3}}=\frac{2 h}{\left\{\left(\vec{x}-\vec{x}^{\prime}\right)^{2}+4 h^{2}\right\}^{3 / 2}}+\left\{\frac{z(\vec{x})+z\left(\vec{x}^{\prime}\right)}{\left|r(\vec{x})-r\left(\vec{x}^{\prime}\right)\right|^{3}}-\frac{2 h}{\left\{\left(\vec{x}-\vec{x}^{\prime}\right)^{2}+4 h^{2}\right\}^{3 / 2}}\right\}
$$

and, like in the case of the whole space, we make the replacement

$$
\partial \Omega_{3} / \partial t=E_{3}\{\Omega, z\}-B_{3}\{z\} \partial \Omega / \partial t
$$


in the term which does not contain the interface gradient. We obtain

$$
\frac{\partial \Omega_{j}}{\partial t}+\alpha \int \frac{2 h}{\left\{\left(\vec{x}-\vec{x}^{\prime}\right)^{2}+4 h^{2}\right\}^{3 / 2}} \frac{\partial \Omega_{j}}{\partial t}\left(\vec{x}^{\prime}\right) d \vec{x}^{\prime}=-\alpha A_{j}\{z\} \frac{\partial \Omega}{\partial t}+F_{j}\{\Omega, z\}
$$

for $j=1,2$ and

$$
\partial \Omega_{3} / \partial t+A_{3}\{z\} \partial \Omega / \partial t=F_{3}\{\Omega, z\}
$$

For $j=1,2$

$$
F_{j}\{\Omega, z\}=E_{j}\{\Omega, z\}+\int\left(\frac{x_{j}-x_{j}^{\prime}}{\left|r(\vec{x})-r\left(\vec{x}^{\prime}\right)\right|^{3}}-\frac{x_{j}-x_{j}^{\prime}}{\left|r(\vec{x})-\bar{r}\left(\vec{x}^{\prime}\right)\right|^{3}}\right) E_{3}\{\Omega, z\}\left(\vec{x}^{\prime}\right) d \vec{x}^{\prime},
$$

$$
\begin{aligned}
& A_{j}\{z\} \frac{\partial \Omega}{\partial t}=-\frac{1}{4 \pi} \int\left(\frac{1}{\left|r(\vec{x})-r\left(\vec{x}^{\prime}\right)\right|^{3}}-\frac{1}{\left|r(\vec{x})-\bar{r}\left(\vec{x}^{\prime}\right)\right|^{3}}\right) \\
& \quad \times\left\{\frac{\partial z}{\partial x_{j}}(\vec{x})\left(\left(x_{j}-x_{\tilde{j}}^{\prime}\right) \frac{\partial \Omega_{j}}{\partial t}\left(\vec{x}^{\prime}\right)-\left(x_{j}-x_{j}^{\prime}\right) \frac{\partial \Omega_{j}}{\partial t}\left(\vec{x}^{\prime}\right)\right)\right. \\
& \left.\quad+\left(x_{j}-x_{j}^{\prime}\right) B_{3}\{z\} \frac{\partial \Omega}{\partial t}\left(\vec{x}^{\prime}\right)\right\}+\frac{z(\vec{x})-z\left(\vec{x}^{\prime}\right)}{\left|r(\vec{x})-r\left(\vec{x}^{\prime}\right)\right|^{3}} \frac{\partial \Omega_{j}}{\partial t}\left(\vec{x}^{\prime}\right) \\
& +\left\{\frac{z(\vec{x})+z\left(\vec{x}^{\prime}\right)}{\left|r(\vec{x})-\bar{r}\left(\vec{x}^{\prime}\right)\right|^{3}}-\frac{2 h}{\left\{\left(\vec{x}-\vec{x}^{\prime}\right)^{2}+4 h^{2}\right\}^{3 / 2}}\right\} \frac{\partial \Omega_{j}}{\partial t}\left(\vec{x}^{\prime}\right) d \vec{x}^{\prime}
\end{aligned}
$$

and

$$
\begin{gathered}
A_{3}\{z\} \partial \Omega / \partial t=B_{3}\{z\} \partial \Omega / \partial t, \\
F_{3}\{\Omega, z\}=E_{3}\{\Omega, z\} .
\end{gathered}
$$

The linear operator acting on $\partial \Omega / \partial t$ in the left-hand side of (3.36) is inverted by taking the Fourier transform of the equation (see Appendix). We finally obtain

PROPOSITION 3.3. When the Rayleigh-Taylor problem is considered in the halfspace $z>0$ and the interface can be resolved in the form $z=z(\vec{x}, t) \equiv h+\eta(\vec{x}, t)$, the equations of motion read

$$
\begin{aligned}
& \text { (a) } \frac{\partial z}{\partial t}-V_{3}+\frac{\partial z}{\partial x_{1}} V_{1}+\frac{\partial z}{\partial x_{2}} V_{2}=0 \\
& \text { (b) } \frac{\partial \Omega_{j}}{\partial t}+\alpha \mathcal{G}(\vec{x}) *\left(A_{j}\{z\} \frac{\partial \Omega_{j}}{\partial t}\right)=\mathcal{G}(\vec{x}) * F_{j}\{\Omega, z\}, \quad j=1,2 \\
& \text { (c) } \frac{\partial \Omega_{3}}{\partial t}+A_{3}\{z\} \frac{\partial \Omega}{\partial t}=F_{3}\{\Omega, z\}
\end{aligned}
$$

where

$$
\mathcal{G}(\vec{x})=\int_{0}^{\infty} \frac{2 h k J_{0}(k|x|)}{1+\alpha e^{-2 h k}} d k
$$

belongs to $L^{1}\left(R^{2}\right)$ (see Appendix) and where the operators $A$ and $F$ are defined in (3.38)-(3.41).

4. A Cauchy-Kowalewski theorem for a nonlinear problem of Fredholm type. Let $B=\left\{B_{s}\right\}$ for $0<s \leq s_{0}$ be a scale of decreasing Banach spaces, i.e. for any $0<s^{\prime}<s \leq s_{0}$

$$
B_{s} \subset B_{s^{\prime}}, \quad\|\|_{s^{\prime}} \leq\|\|_{s} .
$$


Consider in $B^{2}=\left\{B_{s}^{2}\right\}$ the initial value problem for $X=\left(X_{1}, X_{2}\right)$ of the form $(\alpha \in R)$

$$
\begin{gathered}
\partial X_{1} / \partial t+\alpha A\left(t, X_{2}\right) \partial X_{1} / \partial t=F_{1}(t, X), \quad \partial X_{2} / \partial t=F_{2}(t, X) \\
X_{1}(0)=X_{1}^{(0)}, \quad X_{2}(0)=X_{2}^{(0)} .
\end{gathered}
$$

We assume that $X_{1}^{(0)}$ and $X_{2}^{(0)}$ are in $B_{s_{0}}$ with $\left\|X_{1}^{(0)}\right\|_{s}<R_{1}^{(0)}$ and $\left\|X_{2}^{(0)}\right\|_{s}<R_{2}^{(0)}$. We define

$$
\mathcal{E}_{s}\left(R_{1}, R_{2}\right)=\left\{X \in B_{s}^{2},\left\|X_{1}\right\|_{s}<R_{1},\left\|X_{2}\right\|_{s}<R_{2}\right\}
$$

and assume the following hypothesis on $F=\left(F_{1}, F_{2}\right)$ and $A$. For some positive numbers $R_{0}>2 R_{0}^{(1)}, R_{2}>2 R_{0}^{(2)}$ and $T$, let $(t, X) \rightarrow F(t, X)$ be a continuous mapping of $[-T, T] \times \mathcal{E}_{s}\left(R_{1}, R_{2}\right)$ into $B_{s}^{2}$, satisfying for any $0<s^{\prime}<s \leq s_{0}$ and all $X, \tilde{X} \in \mathcal{E}_{s}\left(R_{1}, R_{2}\right)$

$$
\|F(t, X)-F(t, \tilde{X})\|_{s^{\prime}} \leq \frac{C_{F}}{s-s^{\prime}}\|X-\tilde{X}\|_{s}
$$

where $C_{F}$ is a constant independent of $t, X, \tilde{X}, s, s^{\prime}$, but possibly dependent on $R_{1}$ and $R_{2}$. In addition, for $0<s<s_{0}$

$$
\sup _{|t|<T}\left\|F\left(t, X^{(0)}\right)\right\|_{s} \leq \frac{M}{s_{0}-s} .
$$

In (H1) and (H2), the norm in $B_{s}^{2}$ is defined as

$$
\|Y\|_{s}=\left\|Y_{1}\right\|_{s}+\left\|Y_{2}\right\|_{s} \text { for } Y=\left(Y_{1}, Y_{2}\right) \in B_{s}^{2} .
$$

Let $A\left(t, X_{2}\right) Y_{1}$ be a continuous mapping of $\left\{X_{2}, Y_{1} \in B_{s}\right.$, with $\left.\left\|X_{2}\right\|<R_{2}\right\}$ into $B_{s}$, linear in $Y_{1}$ and satisfying, for any $0<s \leq s_{0}$ and all $X_{2}, Y_{1}, \tilde{X}_{2}, \tilde{Y}_{1} \in B_{s}$, with $\left\|X_{2}\right\|_{s}<R_{2}$ and $\left\|\tilde{X}_{2}\right\|_{s}<R_{2}$,

$$
\begin{gathered}
\sup _{|t|<T}\left\|A\left(t, X_{2}\right) Y_{1}-A\left(t, X_{2}\right) \tilde{Y}_{1}\right\|_{s} \leq C_{A}\left\|Y_{1}-\tilde{Y}_{1}\right\|_{s}, \\
\sup _{|t|<t}\left\|A\left(t, X_{2}\right) Y_{1}-A\left(t, \tilde{X}_{2}\right) Y_{1}\right\|_{s} \leq C_{A}^{\prime}\left\|Y_{1}\right\|_{s}\left\|X_{2}-\tilde{X}_{2}\right\|_{s},
\end{gathered}
$$

where $C_{A}$ and $C_{A}^{\prime}$ are constants independent of $t, X_{2}, \tilde{X}_{2}, Y_{1}, \tilde{Y}_{2}, s$ but possibly dependent on $R_{2}$.

THEOREM 4.1. If there exists a constant $c_{*}<1$ such that $(\mathrm{H} 1)$ and $(\mathrm{H} 4)$ are satisfied with $|\alpha| C_{A}<c_{*}$, then there is a positive constant $a$ and a unique function $X(t)$ which, for every $s \in\left(0, s_{0}\right)$, is continuously differentiable in $\left(-a\left(s_{0}-s\right)\right.$, $\left.a\left(s_{0}-s\right)\right)$, valued in $B_{s}^{2}$ with

$$
\sup _{|t|<a\left(s_{0}-s\right)}\left\|X_{1}(t)\right\|_{s}<R_{1} \quad \text { and } \sup _{|t|<a\left(s_{0}-s\right)}\left\|X_{2}(t)\right\|_{s}<R_{2}
$$

and satisfies (4.1).

REMARK 4.1. If (H3) can be replaced by

$$
\left\|A\left(t, X_{2}\right) Y_{1}-A\left(t, X_{2}\right) \tilde{Y}_{1}\right\|_{s} \leq \tilde{C}_{A}\left\|X_{2}\right\|_{s}\left\|Y_{1}-\tilde{Y}_{1}\right\|_{s}
$$


the smallness condition on the constant $C_{A}$ is replaced by a constraint on the initial data, namely $|\alpha| \tilde{C}_{A}\left\|X_{2}^{(0)}\right\|_{s}<1$, so that the coefficient of $\partial X_{1} / \partial t$ is not 0 .

REMARK 4.2 If, in addition to (H1)-(H4) with $t$ complex, $F$ and $A$ satisfy the assumption:

If $0<s^{\prime}<s<s_{0}$, and $X$ holomorphic for $t \in C,|t|<T$ valued in $B_{s}$, then $t \rightarrow F(t, X(t))$ and $t \rightarrow A\left(t, X_{2}(t)\right) Y_{1}(t)$ are holomorphic functions for $|t|<T$ valued in $B_{s}^{\prime}$ and $B_{s}$, respectively, then $X$ is a holomorphic function of $t$ with values in $\left(B_{s}\right)^{2}$.

Proof of Theorem 4.1. Baouendi and Goulaouic's proof $[\mathbf{1 4}]$ of the CauchyKowalewski Theorem as formulated by Nishida [13] for (4.1) with $A=0$ is easily extended to equations of Fredholm type.

For every $a>0, E_{a}$ denotes the Banach space of functions $u(t)$ which for every $s \in\left(0, s_{0}\right)$ are continuous for $|t|<a\left(s_{0}-s\right)$, valued in $B_{s}$ and satisfy

$$
\|u\|_{a}=\sup _{|t|<a\left(s_{0}-s\right)}\|u(t)\|_{s}\left(s_{0}-s\right)\left(1-\frac{|t|}{a\left(s_{0}-s\right)}\right)^{1 / 2}<\infty .
$$

The space $E_{a}$ is equipped with the norm \|\|$\|_{a}$.

Denoting

$$
Y=\left(Y_{1}, Y_{2}\right)=d X / d t
$$

one has

$$
X=\not Y \equiv\left(X_{1}^{(0)}+\int_{0}^{t} Y_{1}(\tau) d \tau, X_{2}^{(0)}+\int_{0}^{t} Y_{2}(\tau) d \tau\right)
$$

and (4.1) is rewritten as

$$
Y_{1}+\alpha A(t, \not \nvdash Y) Y_{1}=F_{1}(t, \not H Y), \quad Y_{2}=F_{2}(t, \not H Y) .
$$

Straightforward adaptation of Lemmas 1-3 of $[\mathbf{1 4}]$ to the case of nonzero initial data leads to

LEMMA 4.1. Let $a>0, s \in\left(0, s_{0}\right)$ and $|t|<a\left(s_{0}-s\right)$. If $X \in\left(E_{a}\right)^{2}$, one has the following properties:

$$
\text { (i) }\|\not H Y\|_{s} \leq\left\|X^{(0)}\right\|_{s}+2 a\|\| Y\|\|_{a} .
$$

$$
\text { (ii) } \int_{0}^{t} \frac{\|Y(\tau)\|_{s}}{s(\tau)-s} d \tau \leq 8 a \frac{\|Y \mid\|_{a}}{s_{0}-s} \sqrt{\frac{a\left(s_{0}-s\right)}{a\left(s_{0}-s\right)-|t|}}
$$

with $s(\tau)=\frac{1}{2}(1+s-|\tau| / a)$.

(iii) If $Y \in\left(E_{a}\right)^{2}$ with $\left\|Y_{1}\right\|_{a}<R_{1} / 8 a,\left\|Y_{2}\right\|_{a}<R_{2} / 8 a$ and $\left.\tilde{Y} \in(E)_{2 a}\right)^{2}$ with $\left\|\tilde{Y}_{1}\right\|_{2 a}<R_{1} / 16 a$ and $\left\|\tilde{Y}_{2}\right\|_{2 a}<R_{2} / 16 a$, then, under hypothesis (H1),

$$
\|F(t, \not \nvdash Y(t))-F(t, \not \alpha \tilde{Y}(t))\|_{s} \leq C \int_{0}^{t} \frac{\|Y(\tau)-\tilde{Y}(\tau)\|_{s(\tau)}}{s-s(\tau)} d \tau,
$$

where $s(\tau)$ is any continuous function on $(0, t)$ satisfying $s<s(\tau) \leq \frac{1}{2}\left(s+s_{0}-|\tau| / a\right)$. $\forall Y(t)$ is defined in (4.4) and

$$
\tilde{X}(t)=\not H \tilde{Y}(t) \equiv\left(X_{1}^{(0)}+\int_{0}^{t} \tilde{Y}_{1}(\tau) d \tau, X_{2}^{(0)}+\int_{0}^{t} \tilde{Y}_{2}(\tau) d \tau\right) .
$$


(4.1) is rewritten in the form

$$
Y=G(Y)
$$

with

$$
G_{1}(Y)=F_{1}(t, \not Y Y)=\alpha A\left(t, \not H F_{2}(t, \not H Y)\right) Y_{1}, \quad G_{2}(Y)=F_{2}(t, \not H Y) .
$$

Let $b \in(0, T)$. If for $|t|<b\left(s_{0}-s\right)$ and $s \in\left(0, s_{0}\right), Y \in E_{b}$ and $\|\mid Y\| \|_{b}<R / 2 b$, one has

$$
\begin{aligned}
\|G(Y(t))\|_{s} \leq & \| F\left(t, \not \nvdash Y(t)\left\|_{s}+|\alpha|\right\| A\left(t, \not \forall F_{2}(t, \not H Y)\right) Y_{1} \|_{s}\right. \\
\leq & \left\|F(t, \not \nvdash Y(t))-F\left(t, X^{(0)}\right)\right\|_{s}+\left\|F\left(t, X^{(0)}\right)\right\|_{s} \\
& +|\alpha|\left\|A\left(t, \not H F_{2}(t, \not \nvdash Y)\right) Y_{1}\right\|_{s} .
\end{aligned}
$$

Lemma 4.1 and hypotheses (H1)-(H3) imply

$$
\begin{gathered}
\|G(Y(t))\|_{s} \leq \frac{8 b c}{s_{0}-s}\|Y\|\left\|_{b} \sqrt{\frac{b\left(s_{0}-s\right)}{b\left(x_{0}-s\right)-|t|}}+\frac{M}{s_{0}-s}+|\alpha| C_{A}\right\| Y_{1} \|_{s} \\
\|\| G(Y)\left\|_{b} \leq 8 b c\left|\|Y\|\left\|_{b}+M+|\alpha| C_{A} \mid\right\| Y_{1} \|_{b} .\right.\right.
\end{gathered}
$$

This inequality insures that, for $a \in(0, T / 2), Y \in\left(E_{a}\right)^{2}$ with $\left\|Y_{i}\right\|<R_{i} / 8 a$ and $\tilde{Y} \in\left(E_{2 a}\right)^{2}$ with $\left\|\tilde{Y}_{i}\right\|_{2 a}<R_{i} / 16 a(i=1,2), G(Y)$ and $G(\tilde{Y})$ are in $\left(E_{a}\right)^{2}$. Furthermore,

$$
\begin{aligned}
\|G(Y(t))-G(\tilde{Y}(t))\|_{s} \leq & \frac{8 a C}{s_{0}-s}\|Y-\tilde{Y} \mid\| \|_{a} \sqrt{\frac{a\left(s_{0}-s\right)}{a\left(s_{0}-s\right)-|t|}} \\
& +|\alpha| C_{A}\left\|Y_{1}(t)-\tilde{Y}_{1}(t)\right\|_{s} \\
& +|\alpha| C_{A}^{\prime}\left\|Y_{1}(t)\right\|_{s}\left\|\nvdash F_{2}(t, \not Y Y)-\not H F_{2}(t, \not H \tilde{Y})\right\|_{s}
\end{aligned}
$$

with

$$
\begin{aligned}
\left\|\not H F F_{2}(t, \not H Y)-\not H F F_{2}(t, \not{H} \tilde{Y})\right\|_{s} & \leq \int_{0}^{t}\left\|F_{2}(\tau, \not{H} Y(\tau))-F_{2}(\tau, \not{H} \tilde{Y}(\tau))\right\|_{s} d \tau \\
& \leq \int_{0}^{t} C\left(\int_{0}^{\tau} \frac{\left\|Y(\tau)-\tilde{Y}\left(\tau^{\prime}\right)\right\|_{s\left(\tau^{\prime}\right)}}{s-s\left(\tau^{\prime}\right)} d \tau^{\prime}\right) d \tau \\
& \leq C 8 a \frac{\|Y-\tilde{Y}\|_{a}}{s_{0}-s} \int_{0}^{t} \sqrt{\frac{a\left(s_{0}-s\right)}{a\left(s_{0}-s\right)-|\tau|}} d \tau \\
& \leq 16 C a^{2}\||| Y-\tilde{Y}\|_{a} .
\end{aligned}
$$

Substituting in (4.15) we obtain

$$
\|G(Y)-G(\tilde{Y})\|\|\leq K \mid\| Y-\tilde{Y} \|_{a}
$$

with

$$
K=a\left(8 C+2|\alpha| C_{A}^{\prime} C R_{1}\right)+|\alpha| C_{A} .
$$

The constant $K$ will be smaller than 1 if $|\alpha| C_{A}<C<1$ and

$$
a<a_{*}=\left(1-C_{*}\right) /\left(8 C+2|\alpha| C_{A}^{\prime} C R_{1}\right) .
$$


Let $E$ denote the closure of $E_{a}$ of the ball

$$
\beta_{2 a}=\left\{Y \in E_{2 a},\left\|Y_{i}\right\|_{2 a}<R_{i} / 16 a, i=1,2\right\} .
$$

$E$ is a complete metric space contained in $\left\{Y \in E_{a},\left\|Y_{i}\right\|_{a}<R_{i} / 16 a(i=1,2)\right\}$. Under the conditions $|\alpha| C_{A}<C_{*}<1$ and $a<\inf \left(T / 2, a_{*}, a_{* *}\right)$ with

$$
a_{* *}=\inf \left(\frac{\left(1-C_{*}\right) R_{1}}{16\left(C \sup _{i} R_{i}+M\right)}, \frac{R_{2}}{16\left(C \sup _{i} R_{i}+M\right)}\right)
$$

$G$ is a contracting mapping of $E$ into itself. Hence $G$ has a unique fixed point $Y$ in $E$ which is a solution of $(4.1)$.

If (H3) is replaced by $\left(\mathrm{H}^{\prime} 3\right)$, the constant $C_{A}$ is replaced by

$$
C_{A}\left(\left\|X_{2}^{(0)}\right\|_{s}+16 C a^{2}\|Y\|_{a}+a M\right)
$$

in (4.14) and (4.15). The constant $K$ in (3.16) becomes

$$
K=a\left\{8 c+2|\alpha| C\left(C_{A}^{\prime} R_{1}+\tilde{C}_{A} \sup _{i=1,2} R_{i}\right)+|\alpha| M\right\}+|\alpha| C_{A}\left\|X_{2}^{(0)}\right\|_{s} .
$$

The conditions which insure that $G$ is a contracting mapping of $E$ into itself are now $|\alpha| C_{A}\left\|X_{2}^{(0)}\right\|_{s}<C_{*}<1$ and $a<\inf \left(T / 2, a_{*}, a_{* *}\right)$ with

$$
\begin{aligned}
& a_{*}=\frac{\left(1-C_{*}\right)}{8 C+2|\alpha| C\left(C_{A} R_{1}+\tilde{C}_{a} \sup R_{i}\right)+|\alpha| M}, \\
& a_{* *}=\inf \left(\frac{\left(1-C_{*}\right) R_{1}}{16\left(C \sup _{i} R_{i}+M\right)\left(1+\alpha \tilde{C}_{A} R_{1}\right)}, \frac{R_{2}}{16\left(C \sup R_{i}+M\right)}\right) .
\end{aligned}
$$

5. Finite time analyticity for the Rayleigh-Taylor problem in the entire space. We look for solutions of systems (2.16), (2.17) and (3.21) such that $\left\{\Omega, y, y_{x}\right\}$ in two dimensions and $\{\vec{\Omega}, z, \nabla z\}$ in three dimensions are analytic functions defined in

$$
b_{s}=\left\{(\vec{x}, \vec{\sigma}), x \in R^{d},|\vec{\sigma}|<s\right\}, \quad d=1 \text { or } 2,
$$

with the additional condition that $\Omega$ and $y_{x}$ or $\nabla z$ belong to $L^{2}\left(R^{d}+i \vec{\sigma}\right)(|\vec{\sigma}|<$ s. $d=1,2)$. We defined the norms

$$
\begin{aligned}
& |u|_{s}=\sup _{x+i \sigma \in b_{s}}|u(x+i \sigma)|, \\
& \|u\|_{s}=|u|_{s}+\sup _{\substack{x+i \sigma \in b_{s} \\
x+i \sigma \in b_{s}}} \frac{\left|u(x+i \sigma)-u\left(x^{\prime}+i \sigma\right)\right|}{\left|x-x^{\prime}\right|^{\alpha}}, \quad 0<\alpha<1, \\
& \|u\|_{L_{s}^{2}}^{2}=\sup _{|\sigma|<s} \int_{R^{d}}|u(x+i \sigma)|^{2} d x, \quad\left|\|u \mid\|_{s}=\|u\|_{s}+\|u\|_{L_{s}^{2} .}\right.
\end{aligned}
$$

$B_{s}^{(1)}$ and $B_{s}^{(2)}$ are, respectively, the spaces of analytic functions $u$ defined in $b_{s}$ such that $\|u\|_{s}$ and \|\|$u \|_{s}$ are bounded.

a. Two dimensional flows. For $\left|\operatorname{Im} y_{x}\right|_{s}<1$ (so that the denominator is not cancelled in (5.3)), $V$ and $A$ are continued analytically to the strip $b_{s}$ in the form

$$
V_{1}\{\Omega, y\}(x+i \sigma)=-\frac{1}{2 \pi} \int \frac{y(x+i \sigma)-y\left(x^{\prime}+i \sigma\right)}{\left(x-x^{\prime}\right)^{2}+\left(y(x+i \sigma)-y\left(x^{\prime}+i \sigma\right)\right)^{2}} \Omega\left(x^{\prime}+i \sigma\right) d x^{\prime},
$$


(5.4) $V_{2}\{\Omega, y\}(x+i \sigma)=\frac{1}{2 \pi} \int \frac{x-x^{\prime}}{\left(x-x^{\prime}\right)^{2}+\left(y(x+i \sigma)-y\left(x^{\prime}+i \sigma\right)\right)^{2}} \Omega\left(x^{\prime}+i \sigma\right) d x^{\prime}$,

$$
\begin{aligned}
& \left(A\{y\} \frac{\partial \Omega}{\partial t}\right)(x+i \sigma) \\
& \quad=\frac{-1}{2 \pi} \int \frac{y(x+i \sigma)-y\left(x^{\prime}+i \sigma\right)-y_{x}(x+i \sigma)\left(x-x^{\prime}\right)}{\left(x-x^{\prime}\right)^{2}+\left(y(x+i \sigma)-y\left(x^{\prime}+i \sigma\right)\right)^{2}} \Omega\left(x^{\prime}+i \sigma\right) d x^{\prime}
\end{aligned}
$$

$V_{1}, V_{2}$ and $A$ satisfy

Proposition 5.1. If $y \in B_{s}^{(1)}$ and $y_{x}, \Omega, \xi \in B_{s}^{(2)}$ with $\left|\operatorname{Im} y_{x}\right|_{s}<\kappa<$ 1, $\left\|\mid y_{x}\right\| \|_{s}<R_{2}$, and similar conditions for $\tilde{y}, \tilde{y} x, \tilde{\Omega}, \tilde{\xi}$, then the analytic continuation of $V$ and and $A$ to complex values of the space variable satisfies

$\left\|V_{1}\{\Omega, y\}-V_{1}\{\tilde{\Omega}, \tilde{y}\} \mid\right\|_{s} \leq C\left(1+R_{2}^{2}\right)\left(\left\|y_{x}\left|\left\|_{s}\right\|\right| \Omega-\tilde{\Omega}\right\|_{s}+\left.\|\tilde{\Omega}\|\right|_{s}\left\|y_{x}-\tilde{y}_{x} \mid\right\|_{s}\right)$,

$$
\begin{aligned}
& || V_{2}\{\Omega, y\}-V_{2}\{\tilde{\Omega}, \tilde{y}\} \mid \|_{s} \leq C\left(1+R_{2}^{2}\right)\left(|||\Omega-\tilde{\Omega}|\left\|_{s}+|| \tilde{\Omega}\right\| \|_{s}|| y_{x}-\left.\tilde{y}_{x}||\right|_{s}\right) \\
& \|A\{y\} \xi-A\{y\} \tilde{\xi}\|_{s} \leq C\left|\left\|y _ { x } \left|\left\|_{s}\left(1+R_{2}^{2}\right)|| \mid \xi-\tilde{\xi}\right\|_{s}\right.\right.\right. \\
& \left\|A\{y\} \xi-A\{\tilde{y}\} \xi\left|\left\|_{s} \leq C\left(1+R_{2}^{2}\right)\right\|\right|\left|\xi\left\|_{s}\right\|\right| y_{x}-\tilde{y}_{x}\right\|_{s},
\end{aligned}
$$

where $C$ is a numerical constant.

ProOF. Inequalities (5.6) and (5.7) are established in [12] with an additional term $|y-\tilde{y}|_{s}$ in the right-hand side. One can actually avoid the introduction of this term by modifying in the estimate of $\left|V_{1}\{\Omega, y\}-V_{1}\{\tilde{\Omega}, \tilde{y}\}\right|_{L_{s}^{2}}$ the contribution of the term

$$
b=\int d x\left(\int d x^{\prime} \theta_{2}\left(\left|x-x^{\prime}\right|\right) \frac{\left(\Omega\left(x^{\prime}+i \sigma\right)-\tilde{\Omega}\left(x^{\prime}+i \sigma\right)\right)\left(y(x+i \sigma)-y\left(x^{\prime}+i \sigma\right)\right)}{\left(x-x^{\prime}\right)^{2}+\left(y(x+i \sigma)-y\left(x^{\prime}+i \sigma\right)\right)^{2}}\right)^{2}
$$

where $\theta_{2}(|x|)=1-\theta_{1}(|x|)$ and $\theta_{1}$ is a smooth function with compact support equal to 1 in a neighborhood of $x=0$. We write

$$
\begin{aligned}
|b| & \leq|\Omega-\tilde{\Omega}|_{L_{s}^{2}}^{2} \int d x\left(\int d x^{\prime} \frac{\theta_{2}^{2}\left(\left|x-x^{\prime}\right|\right)}{\left|x-x^{\prime}\right|^{2}} \int_{0}^{1}\left|y_{x}\left(\lambda x+(1-\lambda) x^{\prime}\right)\right|^{2} d \lambda\right) \\
& \leq|\Omega-\tilde{\Omega}|_{L_{s}^{2}}^{2} \int d x \frac{\theta_{2}^{2}(|x|)}{|x|^{2}} \int d x^{\prime} \int_{0}^{1} d \lambda\left|y_{x}\left(\lambda x+x^{\prime}\right)\right|^{2} d \lambda \\
& \leq C|\Omega-\tilde{\Omega}|_{L_{s}^{2}}^{2}\left|y_{x}\right|_{L_{s}^{2}}^{2} .
\end{aligned}
$$

The inequalities (5.8) and (5.9) are easily obtained by noticing that

$$
A\{y\} \xi=V_{1}\{y, \xi\}+y_{x} V_{2}\{y, \xi\}
$$

and using (5.6) and (5.7).

Using that an analytic function in $b_{s}$ satisfies

$$
\||\nabla f|\|_{s^{\prime}} \leq \frac{1}{s-s^{\prime}}\left|\|f \mid\|_{s}\right.
$$


for $s^{\prime}<s$, one easily checks that the system of equations

$$
\begin{gathered}
\frac{\partial \Omega}{\partial t}+\alpha A\{y\} \frac{\partial \Omega}{\partial t}=F, \quad \frac{\partial y}{\partial t}-V_{2}+y_{x} V_{1}=0 \\
\frac{\partial}{\partial t} y_{x}-\frac{\partial V_{2}}{\partial x}+\frac{\partial}{\partial x}\left(y_{x} V_{1}\right)=0
\end{gathered}
$$

with initial conditions

$$
\Omega(x, 0)=\Omega_{0}(x) \in B_{s}^{(2)}, \quad y(x, 0)=y_{0}(x) \in B_{s}^{(1)}, \quad y_{x}(x, 0)=y_{x, 0}(x) \in B_{s}^{(2)},
$$

satisfies the hypothesis of Theorem 4.1. Since only $\left\|\left|y_{x}\right|\right\|_{s}$ (and not $\||y|\| \|_{s}$ ) appears in the right-hand side of (5.8), the condition for existence of a solution will be $\left.|\alpha|||\left|y_{x, 0}\right|\right|_{s}$ sufficiently small. We thus obtain

THEOREM 5.1. There exists a constant $k_{0}$, such that for initial conditions $\left(y_{0}, d y_{0} / d x, \Omega_{0}\right)$ whose analytic continuation belongs to $B_{s}^{(1)} \times B_{s}^{(2)} \times B_{s}^{(2)}$ with the conditions $\left|\operatorname{Im}\left(d y_{0} / d x\right)\right|_{s_{0}}<\kappa<1$ and $\left.|\alpha|||\left|d y_{0} / d x\right|\right|_{s_{0}}<k_{0}$, there exists a constant $a$, such that for $|t|<a\left(s_{0}-s\right)$, the system (5.14) has a unique solution $(y, d y / d x, \Omega)$ which is an analytic function of $t$ with value in $B_{s}^{(1)} \times B_{s}^{(2)} \times B_{s}^{(2)}$.

b. Three-dimensional flows. For $|\nabla z|_{s}<\kappa<1$, the operators entering in (3.21) are analytically continued to the strip $b_{s}$ according to the following procedure: if

$$
P(\vec{x})=\int_{R^{2}} P\left(\vec{x}, \vec{x}^{\prime}\right) d \vec{x}^{\prime}
$$

then

$$
P(\vec{x}+i \vec{\sigma})=\int_{R^{2}} P\left(\vec{x}+i \vec{\sigma}, \vec{x}^{\prime}+i \vec{\sigma}\right) d \vec{x}^{\prime}
$$

One has

PROPOSITION 5.2. If $z \in B_{s}^{(1)}$ and $\nabla z, \Omega, \xi \in B_{s}^{(2)}$ with $|\operatorname{Im} \nabla z|_{s}<K<$ $1,|| \nabla z \mid \|_{s}<R_{2}$ and similar conditions for $\tilde{z}, \nabla \tilde{z}, \tilde{\Omega}, \tilde{\xi}$, then the analytic continuation to $b_{s}$ of the operators defined in (3.24), (3.13), (3.16)-(3.18) and (3.23) satisfies $\left(0<s^{\prime}<s\right)$

$$
\begin{aligned}
& || V\{\Omega, z\}-V\{\tilde{\Omega}, \tilde{z}\} \mid \|_{s} \leq C\left(R_{2}\right)\left(|||\Omega-\tilde{\Omega}|\left\|_{s}+\right\|\left|\tilde{\Omega}\|\|_{s}\right||| \nabla z-\nabla \tilde{z}|\||_{s}\right), \\
& \||S\{\Omega, z\}-S\{\tilde{\Omega}, \tilde{z}\}|\|_{s^{\prime}} \leq \frac{C\left(R_{2}\right)}{s-s^{\prime}}\left(|||\Omega-\tilde{\Omega}|\left\|_{s}+\right\| \tilde{\Omega}\left|\left\|_{s}||\left|\nabla_{z}-\tilde{z}\right|\right\|_{s}\right),\right. \\
& |||I\{\Omega, z\}-I\{\tilde{\Omega}, \tilde{z}\}| \|_{s^{\prime}} \leq \frac{C\left(R_{2}\right)}{s-s^{\prime}}\left(|||\Omega-\tilde{\Omega}|\left\|_{2}+\right\|\left|\tilde{\Omega}\|\|_{s}\right||| \nabla z-\nabla \tilde{z}|\||_{s}\right), \\
& |||J\{\Omega, z\}-J\{\tilde{\Omega}, \tilde{z}\}| \|_{s^{\prime}} \leq \frac{C\left(R_{2}\right)}{s-s^{\prime}}\left(|||\Omega-\tilde{\Omega}|\left\|_{s}+|| \tilde{\Omega}|||||| \nabla z-\nabla \tilde{z} \mid\right\|_{s}\right), \\
& \left\|A_{j}\{z\} \xi-A_{j}\{z\} \tilde{\xi}\left|\left\|\leq C\left(R_{2}\right)\left|\left\|\nabla z \left|\|\|_{s}\|\mid \xi-\tilde{\xi}\|_{s},\right.\right.\right.\right.\right.\right. \\
& \left\|\left|A_{j}\{z\} \xi-A_{j}\{\tilde{z}\} \xi\right|\right\| \leq C\left(R_{2}\right)\left|\left\|\xi\left|\left\|_{s}\right\|\right| \nabla z-\nabla \tilde{z}\right\|_{s} .\right.
\end{aligned}
$$


PROOF. One uses the following inequalities proved in $[\mathbf{1 0}]$ :

$$
\begin{aligned}
& \| \int \frac{x_{i}-x_{i}^{\prime}}{\left|r(\vec{x}+i \vec{\sigma})-r\left(\vec{x}^{\prime}+i \vec{\sigma}\right)\right|^{3}} \xi\left(\vec{x}^{\prime}+i \vec{\sigma}\right) d x^{\prime} \\
& \quad-\int \frac{x_{i}-x_{i}^{\prime}}{\left|\tilde{r}(\vec{x}+i \vec{\sigma})-\vec{r}\left(\vec{x}^{\prime}+i \vec{\sigma}\right)\right|^{3}} \tilde{\xi}\left(\vec{x}^{\prime}+i \vec{\sigma}\right) d \vec{x}^{\prime}\|\|_{s} \\
& \quad \leq\left(1+C\left(R_{2}\right)\right)\left\{|||\xi-\tilde{\xi}|\left\|_{s}+\left.R_{2}|| \tilde{\xi}||\right|_{s}|||\nabla z-\nabla \tilde{z}|\right\|_{s}\right\}, \quad i=1,2
\end{aligned}
$$

and

$$
\begin{aligned}
& \|\| \int \frac{z(\vec{x}+i \vec{\sigma})-z\left(\vec{x}^{\prime}+i \vec{\sigma}\right)}{\left|r(\vec{x}+i \vec{\sigma})-r\left(\vec{x}^{\prime}+i \vec{\sigma}\right)\right|^{3}} \xi\left(x^{\prime}+i \sigma\right) d x^{\prime} \\
& \quad-\int \frac{\tilde{z}(\vec{x}+i \vec{\sigma})-\tilde{z}\left(\vec{x}^{\prime}+i \vec{\sigma}\right)}{\left|r(\vec{x}+i \vec{\sigma})-\vec{r}\left(\vec{x}^{\prime}+i \vec{\sigma}\right)\right|^{3}} \tilde{\xi}(x+i \sigma) d x^{\prime} \|_{s} \\
& \quad \leq\left(1+C\left(R_{2}\right)\right)\|\| \nabla z \|_{s}\left(\||| \xi-\tilde{\xi}\|_{s}+\left\||| \xi\left|\left\|_{x} \mid\right\| \nabla z-\nabla \tilde{z} \|_{s}\right),\right.\right.
\end{aligned}
$$

where

$$
(\vec{x}+i \vec{\sigma})=\left(x_{1}+i \sigma_{1}, x_{2}+i \sigma_{2}, z(\vec{x}+i \vec{\sigma})\right)
$$

and

$$
r(\vec{x}+i \vec{\sigma})=\left(x_{1}+\sigma_{1}, x_{2}+i \sigma_{2}, \tilde{z}(\vec{x}+i \vec{\sigma})\right)
$$

$C\left(R_{2}\right)$ denotes a constant depending on $R_{2}$.

To prove (5.21), we also use (3.21)(a),

$$
\frac{\partial z}{\partial t}(\vec{x}+i \vec{\sigma})-\frac{\partial z}{\partial t}\left(\vec{x}^{\prime}+i \vec{\sigma}\right)=\left(\vec{x}-\vec{x}^{\prime}\right) \cdot \nabla \int_{0}^{1}\left(V_{3}-\frac{\partial z}{\partial x_{1}} V_{1}-\frac{\partial z}{\partial x_{2}}\right)\left(\lambda \vec{x}+(1-\lambda) \vec{x}^{\prime}\right) d \lambda
$$

and (5.13). This leads to

THEOREM 5.2. There exists a constant $k_{0}$ such that, for initial conditions $\left(z_{0}, \nabla z_{0}, \Omega_{0}\right)$ whose analytic continuation belongs to $B_{s}^{(1)} \times B_{s_{0}}^{(2)} \times B_{s_{0}}^{(2)}$ with the conditions $\left|\operatorname{Im} \nabla z_{0}\right|_{s_{0}}<K<1$ and $\left.|\alpha|||\left|\nabla z_{0}\right|\right|_{s_{0}}<k_{0}$, there exists a constant a such that for $|t|<a\left(s_{0}-s\right)$, the system (3.21) has a unique solution $(z, \nabla z, \Omega)$ which is an analytic function of $t$ with values in $B_{s}^{(1)} \times B_{s}^{(1)} \times B_{s}^{(2)}$.

\section{Local analyticity for the Rayleigh-Taylor problem in half a space.} We shall show in this section that the systems (2.28) and (3.42), for the RayleighTaylor problem in half a space, in two and three dimensions, respectively, satisfy the hypothesis to the Cauchy-Kowalewski Theorem given in $\S 4$. 


$$
A\{y\} \xi(x+i \sigma)=C_{h}\{\eta\} \xi(x+i \sigma)+\int \mathcal{G}\left(x-x^{\prime}\right) C_{h}\{\eta\} \xi\left(x^{\prime}+i \sigma\right) d x^{\prime}
$$

with

$$
\begin{aligned}
& C_{h}\{\eta\} \xi(x+i \sigma) \\
& =\frac{\alpha}{2 \pi} \int\left\{\frac{\eta(x+i \sigma)+\eta\left(x^{\prime}+i \sigma\right)-\eta_{2}(x+i \sigma)\left(x-x^{\prime}\right)}{\left(x-x^{\prime}\right)^{2}+\left(y(x+i \sigma)+y\left(x^{\prime}+i \sigma\right)\right)^{2}}\right. \\
& +2 h \frac{\left(\eta(x+i \sigma)+\eta\left(x^{\prime}+i \sigma\right)\right)\left(y(x+i \sigma)+y\left(x^{\prime}+i \sigma\right)+2 h\right)}{\left[\left(x-x^{\prime}\right)^{2}+\left(y(x+i \sigma)+y\left(x^{\prime}+i \sigma\right)\right)^{2}\right]\left[\left(x-x^{\prime}\right)^{2}+4 h^{2}\right]} \\
& \left.-\frac{\eta(x+i \sigma)-\eta\left(x^{\prime}+i \sigma\right)-\eta_{x}(x+i \sigma)\left(x-x^{\prime}\right)}{\left(x-x^{\prime}\right)^{2}+\left(\eta(x+i \sigma)-\eta\left(x^{\prime}+i \sigma\right)\right)^{2}}\right\} \\
& \times \xi\left(x^{\prime}+i \sigma\right) d x^{\prime}
\end{aligned}
$$

$\mathcal{G}(x)$ given in $(2.31)$ and $y(x+i \sigma)=h+\eta(x+i \sigma)$.

The denominators do not vanish if $\left|\operatorname{Im} y_{x}\right|_{s}<K<1$ and $|\eta|_{s}<\gamma h$, where $\gamma$ is a positive number smaller than one. We shall see that the introduction of the $L^{2}$-norm of the functions $y_{x}$ and $\Omega$ can be avoided because the kernels of $V_{1}$ and $V_{2}$ decrease at least like $\left|x-x^{\prime}\right|^{-2}$ at infinity.

Proposition 6.1. For $\eta, \eta_{x}, \Omega, \xi$ and $\tilde{\eta}, \tilde{\eta}_{x}, \tilde{\Omega}, \tilde{\xi}$ in $B_{s}^{(1)}$ satisfying $\|\eta\|_{s}<$ $\gamma h,\left\|\eta_{x}\right\|<R_{2},\left|\operatorname{Im} y_{x}\right|<K<1$ and similar conditions for $\tilde{\eta}$ and $\tilde{\eta}_{x}$, we have the following estimates:

$$
\begin{aligned}
& \left\|V_{1}\{\Omega, z\}-V_{1}\{\Omega, \tilde{z}\}\right\|_{s} \\
& \quad \leq C\left(\gamma, R_{2}\right)\left\{\left(\frac{\|y\|_{s}}{h}+\frac{\left\|y_{x}\right\|_{s}}{h}\right)\left\|\Omega-\tilde{\Omega}_{s}+\right\| \tilde{\Omega} \|_{s}\left(\frac{\|\eta-\tilde{\eta}\|_{s}}{h}+\frac{\left\|\eta_{x}-\eta_{x}\right\|_{s}}{h}\right)\right\},
\end{aligned}
$$

$\left\|V_{2}\{\Omega, z\}-V_{2}\{\Omega, \tilde{z}\}\right\|_{s} \leq C\left(\gamma, R_{2}\right)\left\{\|\Omega-\tilde{\Omega}\|_{s}+\|\tilde{\Omega}\|_{s}\left(\frac{\|\eta-\eta\|_{s}}{h}+\frac{\left\|\eta_{x}-\tilde{\eta}_{x}\right\|_{s}}{h}\right)\right\}$,

$$
\begin{gathered}
\left\|C_{h}\{\eta\} \xi-C_{h}\{\eta\} \tilde{\xi}\right\|_{s} \leq \alpha C\left(\gamma, R_{2}\right)\left(\gamma+\left\|\eta_{x}\right\|_{s}\right)\|\xi-\tilde{\xi}\|_{s}, \\
\left\|C_{h}\{\eta\} \xi-C_{h}\{\tilde{\eta}\} \xi\right\|_{s} \leq \alpha C\left(\gamma, R_{2}\right)\|\xi\|_{x}\left(\frac{\|\eta-\tilde{\eta}\|_{s}}{h}+\frac{\left\|\eta_{x}-\tilde{\eta}_{x}\right\|_{s}}{h}\right),
\end{gathered}
$$

where $C\left(\gamma, R_{2}\right)$ is uniformly bounded for $\gamma \in\left[0, \gamma_{0}\right]$ with $\gamma_{0}<1$ and $R_{2} \leq R, R$ arbitrary.

Proof. We shall estimate the two terms of $V_{1}$, referred as $V_{1}^{A}$ and $V_{1}^{B}$ separately. We also separate the contribution of short and large distances and write

$$
V_{1}^{A}\{\Omega, y\}=U_{1}^{(1)}\{\Omega, y\}+U_{1}^{(2)}\{\Omega, y\},
$$

where $(i=1,2)$

$$
U_{1}^{(i)}\{\Omega, y\}(x+i \sigma)=-\frac{1}{2 \pi} \int \frac{\left(y(x+i \sigma)-y\left(x^{\prime}+i \sigma\right)\right)\left(x^{\prime}+i \sigma\right)}{\left(x-x^{\prime}\right)^{2}+\left(y(x+i \sigma)-y\left(x^{\prime}+i \sigma\right)\right)^{2}} \theta_{i}\left(\left|x-x^{\prime}\right|\right) d x^{\prime} .
$$


where $C\left(\gamma, R_{2}\right)$ is uniformly bounded for $\gamma \in\left[0, \gamma_{0}\right]$ with $\gamma_{0}<1$ and $R_{2} \leq R, R$ arbitrary.

PROOF. We shall estimate the two terms of $V_{1}$, referred as $V_{1}^{A}$ and $V_{1}^{B}$ separately. We also separate the contribution of short and large distances and write

$$
V_{1}^{A}\{\Omega, y\}=U_{1}^{(1)}\{\Omega, y\}+U_{1}^{(2)}\{\Omega, y\}
$$

where $(i=1,2)$

$$
U_{1}^{(i)}\{\Omega, y\}(x+i \sigma)=-\frac{1}{2 \pi} \int \frac{\left(y(x+i \sigma)-y\left(x^{\prime}+i \sigma\right)\right)\left(x^{\prime}+i \sigma\right)}{\left(x-x^{\prime}\right)^{2}+\left(y(x+i \sigma)-y\left(x^{\prime}+i \sigma\right)\right)^{2}} \theta_{i}\left(\left|x-x^{\prime}\right|\right) d x^{\prime} .
$$

$\theta_{1}$ is a $C^{\infty}$-function with absolute value smaller than one and compact support in $[-2 h, 2 h], \theta_{2}=1-\theta_{1}$. It is shown in $[10]$ that

$$
\left\|U_{1}^{(1)}\{\Omega, y\}-U_{1}^{(1)}\{\tilde{\Omega}, \tilde{y}\}\right\|_{s} \leq C\left(1+R_{2}^{2}\right)\left(\left\|y_{x}\right\|\|\Omega-\tilde{\Omega}\|_{s}+\|\tilde{\Omega}\|_{s}\left\|y_{x}-\tilde{y}_{x}\right\|_{s}\right) .
$$

The introduction of the $L^{2}$-norms of $\Omega$ and $y$ in the estimate of the contribution from large distance is avoided by writing

$$
\left.\mid U_{1}^{(2)}\{\Omega, y\}-U^{(2)}\right)\left.1\{\tilde{\Omega}, \tilde{y}\}\right|_{s} \leq C\left\{\frac{|\eta|_{s}}{h}|\Omega-\tilde{\Omega}|_{s}+|\tilde{\Omega}|_{s}\left(1+R_{2}^{2}\right) \frac{|\eta-\tilde{\eta}|_{s}}{h}\right\}
$$

and

$$
\left|\nabla U^{(2)}\{\Omega, y\}-\nabla U^{(2)}\{\tilde{\Omega}, \tilde{y}\}\right|_{s} \leq C\left(1+R_{2}^{2}\right)\left\{\frac{\left|\eta_{x}\right|_{s}}{h}|\Omega-\tilde{\Omega}|_{s}+|\tilde{\Omega}|_{s} \frac{\left|\eta_{x}-\tilde{\eta}_{x}\right|_{s}}{h}\right\} .
$$

To estimate $V_{1}^{\beta}$ we proceed as above and write

$$
V_{1}^{(R)}\{\Omega, y\}=U_{1}^{(1)}\{\Omega, y\}+U_{1}^{(2)}\{\Omega, y\},
$$

where

$$
U_{1}^{(i)}\{\Omega, y\}=\frac{1}{2 \pi} \int \frac{\left(y(x+i \sigma)+y\left(x^{\prime}+i \sigma\right)\right) \Omega\left(x^{\prime}+i \sigma\right)}{\left(x-x^{\prime}\right)^{2}+\left(y(x+i \sigma)+y\left(x^{\prime}+i \sigma\right)\right)^{2}} \theta_{i}\left(\left|x-x^{\prime}\right|\right) d x^{\prime}
$$

with $\delta \Omega=\Omega-\Omega, \delta y=y-\tilde{y}, \delta \eta=\eta-\tilde{\eta}$; we have

$$
\begin{aligned}
& U_{1}^{(i)}\{\Omega, y\}-U_{1}^{(i)}\{\Omega, y\} \\
& =\frac{1}{2 \pi} \int \theta_{i}\left(\left|x-x^{\prime}\right|\right) \frac{y(x+i \sigma)+y\left(x^{\prime}+i \sigma\right) \delta \Omega\left(x^{\prime}+i \sigma\right)}{\left(x-x^{\prime}\right)^{2}+\left(y(x+i \sigma)+y\left(x^{\prime}+i \sigma\right)\right)^{2}} d x^{\prime} \\
& \quad+\int \theta_{i}\left(\left|x-x^{\prime}\right|\right) \frac{\left(\delta y(x+i \sigma)+\delta y\left(x^{\prime}+i \sigma\right)\right) \tilde{\Omega}\left(x^{\prime}+i \sigma\right)}{\left(x-x^{\prime}\right)^{2}+\left(y(x+i \sigma)+y\left(x^{\prime}+i \sigma\right)\right)^{2}} d x^{\prime} \\
& +\int \theta_{i}\left(\left|x-x^{\prime}\right|\right) \frac{\left(\tilde{y}(x+i \sigma)+\tilde{y}\left(x^{\prime}+i \sigma\right)\right)\left(\delta \eta(x+i \sigma)+\delta \eta\left(x^{\prime}+i \sigma\right)\right)}{\left[\left(x-x^{\prime}\right)^{2}+\left(y(x+i \sigma)+y\left(x^{\prime}+i \sigma\right)\right)^{2}\right]} \\
& \quad \times \frac{\left((y+\tilde{y})(x+i \sigma)+(y+\tilde{y})\left(x^{\prime}+i \sigma\right)\right) \tilde{\Omega}\left(x^{\prime}+i \sigma\right)}{\left[\left(x-x^{\prime}\right)^{2}+\left(y(x+i \sigma)+y\left(x^{\prime}+i \sigma\right)\right)^{2}\right]} d x .
\end{aligned}
$$

For $|\eta|_{s}<\gamma h$, the denominator

$$
D_{+}=\left(x-x^{\prime}\right)^{2}+\left(y(x+i \sigma)+y\left(x^{\prime}+i \sigma\right)\right)^{2}
$$


is bounded from below by

$$
\left|D_{+}\right| \geq h^{2}(1-\gamma)^{2}
$$

is the integral containing $\theta_{1}$ and by

$$
\left|D_{+}\right| \geq\left(x-x^{\prime}\right)^{2}-4 \gamma^{2} h^{2}
$$

is the integral containing $\theta_{2}$. Consequently, for $\|\eta\|_{s}<\gamma h$ and $\|\tilde{\eta}\|_{s}<\gamma h$, (6.18)

$$
\left|U_{1}^{(1)}\{\Omega, y\}-U_{1}^{(1)}\{\tilde{\Omega}, \tilde{y}\}\right|_{s} \leq C\left\{\frac{|\delta \Omega|_{s}}{(1-\gamma)^{2}}+|\Omega|_{s} \frac{|\delta \eta|_{s}}{(1-\gamma)^{2} h}+|\Omega|_{s} \frac{|\delta \eta|_{s}}{(1-\gamma)^{4} h}\right\}
$$

and

$$
\begin{aligned}
& \left|U_{1}^{(2)}\{\Omega, y\}-U_{1}^{(2)}\{\tilde{\Omega}, \tilde{y}\}\right|_{s} \\
& \leq C\left\{|\delta \Omega|_{s} h \int \frac{d x}{\left(x-x^{\prime}\right)^{2}-4 \gamma^{2} h^{2}}+|\Omega|_{s}|\delta \eta|_{s} \int \frac{d x^{\prime}}{\left(x-x^{\prime}\right)^{2}-4 \gamma^{2} h^{2}}\right. \\
& \left.\quad+|\Omega|_{s} h^{2}|\delta \eta|_{s} \int_{|x-x|>2 h} \frac{d x^{\prime}}{\left[\left(x-x^{\prime}\right)^{2}-4 \gamma^{2} h^{2}\right]^{2}}\right\} \\
& \leq C\left\{\frac{1}{\gamma} \ln \frac{1+\gamma}{1-\gamma}\left(|\delta \Omega|_{s}+\frac{|\delta \eta|_{s}}{h}|\Omega|_{s}\right)+\frac{1}{\gamma^{3}}\left(\frac{2 \gamma}{1-\gamma^{2}}-\ln \frac{1+\gamma}{1-\gamma}\right) \frac{|\delta \eta|_{s}}{h}|\Omega|_{s}\right\} .
\end{aligned}
$$

The constants

$$
\frac{1}{\gamma} \ln \frac{1+\gamma}{1-\gamma} \text { and } \quad \frac{1}{\gamma^{3}}\left(\frac{2 \gamma}{1-\gamma^{2}}-\ln \frac{1+\gamma}{1-\gamma}\right)
$$

are uniformly bounded for $\gamma \in\left[0, \gamma_{0}\right]$ with $\gamma_{0}<1$.

To estimate the difference

$$
\Delta=\left(V_{1}^{B}\{\Omega, y\}-V_{1}^{B}\{\Omega, y\}\right)(x+i \sigma)-\left(V_{i}^{B}\{\Omega, y\} V_{1}^{B}\{\tilde{\Omega}, \tilde{y}\}\right)\left(x^{\prime}+i \sigma\right),
$$

we first notice that

$$
\pi \Omega(x+i \sigma)=\int_{-\infty}^{+\infty} \frac{2 y(x+i \sigma) \Omega(x+i \sigma)}{(x-q)^{2}+4 y^{2}(x+i \sigma)} d q .
$$

The equality is obvious in the real domain. It extends to the complex domain because of the uniqueness of the analytic continuation. We then compute the difference $\Delta$. Denoting

$$
D_{+}\{y\}(x+i \sigma, q+i \sigma)=(x-q)^{2}+(y(x+i \sigma)+y(q+i \sigma))^{2}
$$

and

$$
D_{0}\{y\}(x+i \sigma, q+i \sigma)=(x-q)^{2}+4 y^{2}(x+i \sigma)^{2}
$$

we write

$$
\Delta=\sum_{i=1}^{2} \Delta_{1}^{(i)}+\Delta_{2}+\sum_{i=1}^{2} \Delta_{3}^{(i)}+\Delta_{4}+\sum_{i=1}^{2} \Delta_{5}^{(i)}+\Delta_{6}
$$


where

$$
\begin{gathered}
\Delta_{1}^{(i)}=\frac{1}{2 \pi} \int \theta_{i}(|x-q|)\left\{\frac{(y(x+i \sigma)+y(q+i \sigma)) \delta \Omega(q+i \sigma)}{D_{+}\{y\}(x+i \sigma, q+i \sigma)}\right. \\
\left.-\frac{2 y(x+i \sigma) \delta \Omega(x+i \sigma)}{D_{0}\{y\}(x+i \sigma, q+i \sigma)}\right\} d q \\
-\frac{1}{2 \pi} \int \theta_{i}(|x-q|)\left\{\frac{\left(y\left(x^{\prime}+i \sigma\right)+y(q+i \sigma)\right) \delta \Omega(q+i \sigma)}{D_{+}\{y\}\left(x^{\prime}+i \sigma, q+i \sigma\right)}\right. \\
\left.-\frac{2 y\left(x^{\prime}+i \sigma\right) \delta \Omega\left(x^{\prime}+i \sigma\right)}{D_{0}\{y\}\left(x^{\prime}+i \sigma, q+i \sigma\right)}\right\} d q
\end{gathered}
$$

$$
\Delta_{2}=-\frac{1}{2 \pi}\left(\delta \Omega(x+i \sigma)-\delta \Omega\left(x^{\prime}+i \sigma\right)\right)
$$$$
\Delta_{3}^{(i)}=\frac{1}{2 \pi} \int \theta_{i}(|x-q|)\left\{\frac{(\delta y(x+i \sigma)+\delta y(q+i \sigma)) \tilde{\Omega}(q+i \sigma)}{D_{+}\{y\}(x+i \sigma, q+i \sigma)}\right.
$$$$
\left.-\frac{2 \delta y(x+i \sigma) \tilde{\Omega}(q+i \sigma)}{D_{0}\{y\}(x+i \sigma, q+i \sigma)}\right\} d q
$$$$
-\frac{1}{2 \pi} \int \theta_{i}(|x-q|)\left\{\frac{\left(\delta y\left(x^{\prime}+i \sigma\right)+\delta y(q+i \sigma)\right) \tilde{\Omega}(q+i \sigma)}{D_{+}\{y\}\left(x^{\prime}+i \sigma, q+i \sigma\right)}\right.
$$$$
\left.-\frac{2 \delta y\left(x^{\prime}+i \sigma\right) \tilde{\Omega}(q+i \sigma)}{D_{0}\{y\}\left(x^{\prime}+i \sigma, q+i \sigma\right)}\right\} d q
$$

$$
\Delta_{4}=\frac{1}{2}\left(\frac{\delta \eta(x+i \sigma)}{y(x+i \sigma)}-\frac{\delta \eta\left(x^{\prime}+i \sigma\right)}{y\left(x^{\prime}+i \sigma\right)}\right)
$$

$\Delta_{5}^{(i)}=\frac{1}{2 \pi} \int \theta_{i}(|x-q|)\left\{\frac{(\tilde{y}(x+i \sigma)+\tilde{y}(q+i \sigma))(\delta \eta(x+i \sigma)+\delta \eta(q+i \sigma))}{D_{+}\{y\}(x+i \sigma, q+i \sigma)}\right.$

$$
\begin{gathered}
\times \frac{((y+\tilde{y})(x+i \sigma)+(y+\tilde{y})(q+i \sigma))}{D_{+}\{\tilde{y}\}(x+i \sigma, q+i \sigma)} \tilde{\Omega}(q+i \sigma) \\
\left.-\frac{8 \tilde{y}(x+i \sigma) \delta \dot{\eta}(x+i \sigma)(y+\tilde{y})(x+i \sigma) \tilde{\Omega}(x+i \sigma)}{D_{0}\{y\}(x+i \sigma, q+i \sigma) D_{0}\{\tilde{y}\}(x+i \sigma, q+i \sigma)}\right\} d q
\end{gathered}
$$

$$
\begin{aligned}
& +\frac{1}{2 \pi} \int \theta_{i}\left(\left|x^{\prime}-q\right|\right)\left\{\frac{\left(\tilde{y}\left(x^{\prime}+i \sigma\right)+\tilde{y}(q+i \sigma)\right)\left(\delta \eta\left(x^{\prime}+i \sigma\right)+\delta \eta(q+i \sigma)\right)}{D_{+}+\{y\}\left(x^{\prime}+i \sigma, q+i \sigma\right)}\right. \\
& \times \frac{\left((y+\tilde{y})\left(x^{\prime}+i \sigma\right)+(y+y)(q+i \sigma)\right)}{D_{+}\{\tilde{y}\}\left(x^{\prime}+i \sigma, q+i \sigma\right)} \tilde{\Omega}(q+i \sigma) \\
& \left.-\frac{8 \tilde{y}\left(x^{\prime}+i \sigma\right) \delta \eta\left(x^{\prime}+i \sigma\right)(y+\tilde{y})(x+i \sigma) \tilde{\Omega}(x+i \sigma)}{D_{0}\{y\}(x+i \sigma, q+i \sigma) D_{0}\{\tilde{y}\}\left(x^{\prime}+i \sigma, q+i \sigma\right)} d q\right\},
\end{aligned}
$$

(6.30) $\Delta_{6}=8 \int\left\{\frac{y(x+i \sigma) \delta \eta(x+i \sigma)(y+\tilde{y})(x+i \sigma) \tilde{\Omega}(x+i \sigma)}{D_{0}\{y\}(x+i \sigma, q+i \sigma) D_{0}\{y\}(x+i \sigma, q+i \sigma)}\right.$

$$
\left.-\frac{\tilde{y}\left(x^{\prime}+i \sigma\right) \delta \eta\left(x^{\prime}+i \sigma\right)(y+\tilde{y})(x+i \sigma) \tilde{\Omega}\left(x^{\prime}+i \sigma\right)}{D_{0}\{y\}\left(x^{\prime}+i \sigma, q+i \sigma\right) D_{0}\{\tilde{y}\}\left(x^{\prime}+i \sigma, q+i \sigma\right)}\right\} d q .
$$


Let us first assume that $\left|x-x^{\prime}\right|<2 h$. In $\Delta_{j}^{(1)}$, we split the integration domain into the ball $\Sigma=\left\{q,|x-q|<2\left|x-x^{\prime}\right|\right\}$ and its complement $R^{2} \backslash \Sigma$. Let $\delta_{j}^{(1)}$ and $\bar{\delta}_{j}^{(1)}$ be the corresponding contribution. We have

$$
\left|\delta_{1}^{(1)}\right|+\left|\delta_{3}^{(1)}\right|+\left|\delta_{5}^{(1)}\right| \leq C\left|x-x^{\prime}\right|^{\alpha}\left(\|\delta \Omega\|_{s}+\|\tilde{\Omega}\|_{s}\|\delta \eta\|_{s} / h\right)
$$

and

$$
\left|\bar{\delta}_{1}^{(1)}\right|+\left|\bar{\delta}_{3}^{(1)}\right|+\left|\bar{\delta}_{5}^{(1)}\right| \leq C\left|x-x^{\prime}\right|^{\alpha}\left(\|\delta \Omega\|_{s}+\|\tilde{\Omega}\|\|\delta \eta\|_{s} / h\right)
$$

On the other hand,

$$
\begin{aligned}
\left|\Delta_{1}^{(2)}\right|+\left|\Delta_{3}^{(2)}\right|+\left|\Delta_{5}^{(2)}\right| \leq\left|x-x^{\prime}\right|^{\alpha}\left(\|\delta \Omega\|_{s}+\frac{\|\delta \eta\|_{s}}{h}\right) \\
\times\left\{\frac{1}{\gamma} \ln \frac{1+\gamma}{1-\gamma}+\frac{1}{\gamma^{3}}\left(\frac{2 \gamma}{1-\gamma^{2}}-\ln \frac{\gamma+1}{1-\gamma}\right)\right. \\
\quad+\frac{1}{\gamma^{4}} \int_{|\mu|>1 / \gamma} \frac{\mu d \mu}{\left(\mu^{2}-1\right)^{3}}+\frac{1}{\gamma^{5-\alpha}} \int_{|\mu|>1 / \gamma} \frac{\mu^{\alpha} d \mu}{\left(\mu^{2}-1\right)^{3}} \\
\left.+\frac{1}{\gamma^{5}} \int_{|\mu|>1 / \gamma} \frac{\mu^{2} d \mu}{\left(\mu^{2}-1\right)^{4}}+\frac{1}{\gamma^{5-\alpha}} \int_{|\mu|>1 / \gamma} \frac{\mu^{2+\alpha} d \mu}{\left(\mu^{2}-1\right)^{4}}\right\} .
\end{aligned}
$$

When $\left|x-x^{\prime}\right|>2 h$, the two terms of $\Delta_{j}^{(i)}(i=1,2, j=1,3,5)$ are bounded separately and we obtain the same upper bounds. On the other hand, we have

$$
\left|\Delta_{2}\right|+\left|\Delta_{4}\right| \leq C\left(\|\delta \Omega\|_{s}+\|\tilde{\Omega}\|\|\delta \eta\|_{s} /(1-\gamma) h\right)\left|x-x^{\prime}\right|^{\alpha} .
$$

Noticing finally that

$$
\Delta_{6}=-\frac{1}{2}\left(\frac{\Omega(x+i \sigma)}{y(x+i \sigma)} \delta \eta(x+i \sigma)-\frac{\Omega(x+i \sigma)}{y\left(x^{\prime}+i \sigma\right)} \delta \eta\left(x^{\prime}+i \sigma\right)\right)
$$

we obtain

$$
\left\|V_{1}^{B}\{\Omega, y\}-V_{1}^{B}\{\tilde{\Omega}, y\}\right\|_{s} \leq C(\gamma)\left\{\|\Omega-\tilde{\Omega}\|_{s}+\frac{\|\eta-\tilde{\eta}\|_{s}}{h}\|\Omega\|_{s}\right\},
$$

where $C(\gamma)$ is uniformly bounded for all $\gamma$ in $\left[0, \gamma_{0}\right]$ with $\gamma_{1}<1$.

Let us now turn to the estimates for $V_{2}$. As previously, we write

$$
V_{2}\{\Omega, y\}=U_{2}^{(1)}\{\Omega, y\}+U_{2}^{(2)}\{\Omega, y\}
$$

with

$$
\begin{aligned}
& U_{2}^{(i)}\{\Omega, y\}(x+i \sigma)=\frac{1}{2 \pi} \int \theta_{i}\left(\left|x-x^{\prime}\right|\right) \\
& \quad \times\left(\frac{x-x^{\prime}}{\left(x-x^{\prime}\right)^{2}+\left(y(x+i \sigma)-y\left(x^{\prime}+i \sigma\right)\right)^{2}}\right. \\
& \left.\quad-\frac{x-x^{\prime}}{\left(x-x^{\prime}\right)^{2}+\left(y(x+i \sigma)+y\left(x^{\prime}+i \sigma\right)\right)^{2}}\right) \Omega\left(x^{\prime}+i \sigma\right) d x^{\prime}
\end{aligned}
$$

Estimating the two terms of $U_{2}^{(1)}$ separately, we get, proceeding as previously

$$
\left\|U_{2}^{(1)}\{\Omega, y\}-U_{2}^{(1)}\{\tilde{\Omega}, \tilde{y}\}\right\|_{s} \leq C(\gamma)\left(\|\delta \Omega\|_{s}+\frac{\|\delta \eta\|_{s}}{h}\|\tilde{\Omega}\|_{s}+\|\delta \eta\|_{s}\|\tilde{\Omega}\|_{s}\right)
$$


where $C(\gamma)$ is uniformly bounded for $\gamma \in\left[0, \gamma_{0}\right], \gamma_{0}<1$. For the contribution at large distance, we have to deal with the two terms of $U^{(2)}$ together in order to avoid the introduction of $L^{2}$-norms:

$$
\begin{aligned}
& U_{2}^{(2)}\{\Omega, y\}(x+i \sigma) \\
& \quad=\frac{1}{2 \pi} \int \theta_{2}\left(\left|x-x^{\prime}\right|\right) \frac{4\left(x-x^{\prime}\right) y(x+i \sigma) y\left(x^{\prime}+i \sigma\right) \Omega\left(x^{\prime}+i \sigma\right)}{D_{+}\{y\}\left(x+i \sigma, x^{\prime}+i \sigma\right) D_{-}\{y\}\left(x+i \sigma, x^{\prime}+i \sigma\right)} d x^{\prime}
\end{aligned}
$$

where

$$
D_{-}\{y\}\left(x+i \sigma, x^{\prime}+i \sigma\right)=\left(x-x^{\prime}\right)^{2}+\left(y(x+i \sigma)-y\left(x^{\prime}+i \sigma\right)\right)^{2},
$$

and $D_{+}\{y\}$ is defined in (6.22). We have

$$
U_{2}^{(2)}\{\Omega, y\}(x+i \sigma)-U_{2}^{(2)}\{\tilde{\Omega}, \tilde{y}\}(x+i \sigma)=\left(d_{1}+d_{2}+d_{3}+d_{4}+d_{5}\right)(x+i \sigma)
$$

with

$$
d_{1}(x+i \sigma)=U^{(2)}\{\delta \Omega, y\}(x+i \sigma)
$$

$$
d_{2}(x+i \sigma)=\frac{1}{2 \pi} \int \theta_{2}\left(\left|x-x^{\prime}\right|\right) \frac{4\left(x-x^{\prime}\right) \delta \eta(x+i \sigma) y\left(x^{\prime}+i \sigma\right) \tilde{\Omega}\left(x^{\prime}+i \sigma\right)}{D_{+}\{y\}\left(x+i \sigma, x^{\prime}+i \sigma\right) D_{-}\{y\}\left(x+i \sigma, x^{\prime}+i \sigma\right)} d x^{\prime}
$$

$$
d_{3}(x+i \sigma)=\frac{1}{2 \pi} \int \theta_{2}\left(\left|x-x^{\prime}\right|\right) \frac{4\left(x-x^{\prime}\right) \tilde{y}(x+i \sigma) \delta \eta\left(x^{\prime}+i \sigma\right) \tilde{\Omega}\left(x^{\prime}+i \sigma\right)}{D_{+}\{y\}\left(x+i \sigma, x^{\prime}+i \sigma\right) D_{-}\{y\}\left(x+i \sigma, x^{\prime}+i \sigma\right)} d x
$$

$$
\begin{gathered}
d_{4}(x+i \sigma)=\frac{1}{2 \pi} \int \theta_{2}\left(\left|x-x^{\prime}\right|\right) \frac{4\left(x-x^{\prime}\right) \tilde{y}(x+i \sigma) \tilde{y}\left(x^{\prime}+i \sigma\right) \tilde{\Omega}\left(x^{\prime}+i \sigma\right)\left(\delta \eta(x+i \sigma)+\delta \eta\left(x^{\prime}+i \sigma\right)\right)}{D_{+}\{y\}\left(x+i \sigma, x^{\prime}+i \sigma\right) D_{+}\{\tilde{y}\}\left(x+i \sigma, x^{\prime}+i \sigma\right)} \\
\times \frac{\left((\eta+\tilde{\eta})(x+i \sigma)+(\eta+\tilde{\eta})\left(x^{\prime}+i \sigma\right)\right)}{D_{-}\{y\}\left(x+i \sigma, x^{\prime}+i \sigma\right)} d x^{\prime}
\end{gathered}
$$

$$
\begin{gathered}
d_{5}(x+i \sigma)=\frac{1}{2 \pi} \int \theta_{2}\left(\left|x-x^{\prime}\right|\right) \frac{4\left(x-x^{\prime}\right) \tilde{y}(x+i \sigma) y\left(x^{\prime}+i \sigma\right) \tilde{\Omega}\left(x^{\prime}+i \sigma\right)\left(\delta \eta(x+i \sigma)-\delta \eta\left(x^{\prime}+i \sigma\right)\right)}{D_{+}\{\tilde{y}\}\left(x+i \sigma, x^{\prime}+i \sigma\right) D_{-}\{y\}\left(x+i \sigma, x^{\prime}+i \sigma\right)} \\
\times \frac{\left((\eta+\tilde{\eta})(x+i \sigma)-(\eta+\tilde{\eta})\left(x^{\prime}+i \sigma\right)\right)}{D_{-}\{\tilde{y}\}\left(x+i \sigma, x^{\prime}+i \sigma\right)} d x .
\end{gathered}
$$

For $\left|\operatorname{Im} y_{x}\right|<K<1$ and $|\eta|_{s}<\gamma h\left(\gamma \leq \gamma_{0}<1\right)$, we have

$$
\left|d_{1}\right|+\left|d_{2}\right|+\left|d_{3}\right| \leq C\left(|\delta \Omega|_{s}+|\Omega|_{s} \frac{|\delta \eta|_{s}}{h}\right) \frac{1}{\gamma^{2}} \ln \left(1-\gamma^{2}\right)
$$

and

$$
\left|d_{4}\right|+\left|d_{5}\right| \leq C|\Omega|_{s} \frac{|\delta \eta|_{s}}{h}\left(\frac{1}{4} \ln \left(1-\gamma^{4}\right)+\frac{1}{\gamma^{2}} \ln \left(1-\gamma^{2}\right)\right)
$$

Thus,

$$
\left|U_{2}^{(2)}\{\Omega, y\}-U_{2}^{(2)}\{\tilde{\Omega}, \tilde{y}\}\right| \leq C(\gamma)\left\{\|\Omega-\tilde{\Omega}\|_{s}+\frac{\|\delta \eta\|_{s}}{h}\|\tilde{\Omega}\|_{s}\right\}
$$


where $C(\gamma)$ is uniformly bounded for $\gamma \in\left[0, \gamma_{0}\right], \gamma_{0}<1$. To estimate the difference $d_{i}(x+i \sigma)-d_{i}\left(x^{\prime}+i \sigma\right)(i=1,2, \ldots, 5)$, we write

$$
\begin{aligned}
& d_{1}(x+i \sigma)=\frac{1}{2 \pi} \int \theta_{2}(|x-q|)\left\{\frac{4(x-q) y(x+i \sigma) y(q+i \sigma) \delta \Omega(q+i \sigma)}{D_{+}\{y\}(x+i \sigma, q+i \sigma) D_{-}\{y\}(x+i \sigma, q+i \sigma)}\right. \\
& \left.-\frac{4(x-q) y^{2}(x+i \sigma) \delta \Omega(x+i \sigma)}{D_{0}\{y\}(x+i \sigma, q+i \sigma)(x-q)^{2}\left(1+y_{x}^{2}(x+i \sigma)\right)}\right\} d q \\
& =\frac{1}{2 \pi} \int 4 \theta_{2}(x-q)\left\{\frac{(x-q) y(x+i \sigma) y(q+i \sigma) \delta \Omega(q+i \sigma)-y(x+i \sigma) \delta \Omega(x+i \sigma)}{D_{+}\{y\}(x+i \sigma, q+i \sigma) D-\{y\}(x+i \sigma, q+i \sigma)}\right\} \\
& +\frac{1}{2 \pi} \int 4 \theta_{2}(|x-q|) \frac{(x-q) y^{2}(x+i \sigma) \delta \Omega(x+i \sigma)}{D_{-}\{y\}(x+i \sigma, q+i \sigma)} \\
& \quad \times\left\{\frac{(y(x+i \sigma)-y(q+i \sigma))(3 y(x+i \sigma)+y(q+i \sigma))}{D_{0}\{y\}(x+i \sigma, q+i \sigma, q+i \sigma) D_{+}\{y\}(x+i \sigma, q+i \sigma)}\right\} d q \\
& +\frac{1}{2 \pi} \int 4 \theta_{2}(|x-q|) \frac{y^{2}(x+i \sigma) \delta \Omega(x+i \sigma)}{(x-q) D_{0}\{y\}(x+i \sigma, q+i \sigma)} \\
& \times\left\{\frac{\left((x-q) y_{x}(x+i \sigma)-(y(x+i \sigma)-y(q+i \sigma))\right)}{D_{-}\{y\}(x+i \sigma, q+i \sigma)}\right. \\
& \left.\times \frac{\left((x-q) y_{x}(x+i \sigma)+y(x+i \sigma)-y(q+i \sigma)\right)}{\left(1+y_{x}^{2}(x+i \sigma)\right)}\right\} d q
\end{aligned}
$$

and similar expressions for $d_{2}, \ldots, d_{5}$.

When estimating $d_{i}(x+i \sigma)-d_{i}\left(x^{\prime}+i \sigma\right)$, we separate, as previously, the contributions $\left|x-x^{\prime}\right|<2 h$ and $\left|x-x^{\prime}\right|>2 h$ and proceed as above. We obtain

$$
\left\|U_{2}^{(2)}\{\Omega, y\}-U_{2}^{(2)}\{\tilde{\Omega}, \tilde{y}\}\right\|_{s} \leq C(\gamma)\left\{\|\Omega-\tilde{\Omega}\|_{s}+\frac{\|\delta \eta\|_{s}}{h}\|\tilde{\Omega}\|_{s}+\left\|\frac{\delta \eta_{x}}{h}\right\|_{s}\|\tilde{\Omega}\|_{s}\right\} .
$$

This concludes the proof of (6.4) and (6.5).

Let us now turn to the operator $A$. Noticing that the kernels $\mathcal{G}$ and $d \mathcal{G} / d x$ belongs to $L^{1}(R+i \sigma)$, it is sufficient to estimate the operator $C_{h}\{\eta\} \xi$. Let us rewrite $C_{h}\{\eta\}$ in the form

$$
\begin{aligned}
& C_{h}\{\eta\}(x+i \sigma)=\frac{\alpha}{2 \pi} \int \frac{\eta(x+i \sigma)+\eta(q+i \sigma)}{D_{+}\{y\}(x+i \sigma, q+i \sigma)} \xi(q+i \sigma) d q \\
& \quad-\int \frac{\eta(x+i \sigma)-\eta\left(x^{\prime}+i \sigma\right)}{D_{-}\{y\}(x+i \sigma, q+i \sigma)} \xi(q+i \sigma) d q \\
& \quad+\frac{\alpha}{2 \pi} \int 2 h \frac{\left(\eta(x+i \sigma)+\eta\left(x^{\prime}+i \sigma\right)\right)\left(2 h+y(x+i \sigma)+y\left(x^{\prime}+i \sigma\right)\right)}{D_{+}\{y\}(x+i \sigma, q+i \sigma)\left((x-q)^{2}+4 h^{2}\right)} \xi(q+i \sigma) d q \\
& \quad+\frac{\alpha}{2 \pi} \int \eta_{x}(x+i \sigma) \frac{4\left(x_{q}\right) y(x+i \sigma) y(q+i \sigma) \xi(q+i \sigma)}{D_{+}\{y\}(x+i \sigma, q+i \sigma) D_{-}\{y\}(x+i \sigma, q+i \sigma)} d q .
\end{aligned}
$$

Using the upper bounds of $V_{1}$ and $V_{2}$ obtained previously, we get, for $\|\eta\|_{s} \leq \gamma h$ and $\left\|\eta_{x}\right\|<R_{2}$,

$$
\begin{gathered}
\left\|C_{h}\{\eta\} \xi-C_{h}\{\tilde{\eta}\} \xi\right\|_{s} \leq \alpha C\left(\gamma, R_{2}\right)\left\{\gamma\|\xi-\tilde{\xi}\|_{s}+\left\|\eta_{x}\right\|_{s}\|\xi-\tilde{\xi}\|_{s}\right\} \\
\left\|C_{h}\{\eta\} \xi-C_{h}\{\tilde{\eta}\} \xi\right\|_{s} \leq \alpha C\left(\gamma, R_{2}\right)\|\xi\|_{s}\left(\frac{\|\eta-\tilde{\eta}\|_{s}}{h}+\frac{\left\|\eta_{x}-\tilde{\eta}_{x}\right\|_{s}}{h}\right)
\end{gathered}
$$

where $C\left(\gamma, R_{2}\right)$ is uniformly bounded for $\gamma \in\left[0, \gamma_{0}\right], \gamma_{0}<1$, and $R_{2} \leq R, R$ arbitrary. 
THEOREM 6.1. There exists a constant $k_{0}$, such that for initial conditions $\left(y_{0}=h+\eta_{0}, d y_{0} / d x, \Omega_{0}\right)$ whose analytic continuation belongs to $\left(B_{s_{0}}^{(1)}\right)^{3}$ with the conditions $\left|\operatorname{Im}\left(d y_{0} / d x\right)\right|_{s}<K<1,\left\|d y_{0} / d x\right\|_{s}<\gamma h, \gamma \leq \gamma_{0}<1$ and $\alpha\left(\gamma+\left\|d y_{0} / d x\right\|_{s}\right)<k_{0}$, there exists a constant a such that, for $|t|<a\left(s_{0}-s\right)$, the system (2.28) has a unique solution $\left(y, y_{x}, \Omega\right)$ which is an analytic function of $t$ with value in $\left(B_{s}^{(1)}\right)^{3}$.

b. Three-dimensional flows. The operators $V$ and $A$ defined in (3.25), (3.39) and (3.40) are analytically continued to the strip $b_{1}=\left\{(\vec{x}+i \vec{\sigma}), \vec{x} \in R^{2},|0|<s\right\}$ by replacing $\vec{x}$ and $\vec{x}^{\prime}$ by $\vec{x}+i \vec{\sigma}$ and $\vec{x}^{\prime}+i \vec{\sigma}$ in the formulas (3.25) and (3.38)-(3.40).

The denominators will not vanish if $|\operatorname{Im} \nabla z|_{s}<K<1$ and $|\eta|_{s}<\gamma h$, where $\gamma$ is a positive number strictly smaller than one.

Proposition 6.2. For $\eta, \nabla \eta, \Omega, \xi$ and $\tilde{\eta}, \nabla \tilde{\eta}, \tilde{\Omega}, \tilde{\xi}$ in $B_{s}^{(1)}$ satisfying $\|\eta\|_{s}<$ $\gamma h,\|\nabla \eta\|<R_{2},|\operatorname{Im}|,\left.\nabla \eta\right|_{s}<K<1$, and similar conditions for $\tilde{\eta}$ and $\nabla \tilde{\eta}$, we have the following estimates $(z=h+\eta, \tilde{z}=h+\tilde{\eta})$ :

$$
\begin{aligned}
&\|V\{\Omega, z\}-V\{\tilde{\Omega}, \tilde{z}\}\|_{s} \leq C\left(\gamma, R_{2}\right)\left(1+\|z\|_{s} / h+\|\nabla \eta\|_{s} / h\|\Omega-\tilde{\Omega}\|_{s}\right. \\
&+\|\tilde{\Omega}\|_{s}\left\{\|\eta-\tilde{\eta}\|_{s} / h+\|\nabla(\eta-\tilde{\eta})\|_{s} / h\right\} \\
&\left\|A_{j}\{z\} \xi-A_{j}\{z\} \tilde{\xi}\right\|_{s} \leq C\left(\gamma, R_{2}\right)\left(\gamma+\|\nabla \eta\|_{s}\right)\|\xi-\tilde{\xi}\|_{s} \\
&\left\|A_{j}\{z\} \xi-A_{j}\{\tilde{z}\} \xi\right\|_{s} \leq C\left(\gamma, R_{2}\right)\|\xi\|_{s}\left(\|\eta-\tilde{\eta}\|_{s} / h+\|\nabla(\eta-\tilde{\eta})\|_{s} / h\right)
\end{aligned}
$$

Let us define

$$
\begin{gathered}
D_{ \pm}\{z\}\left(\vec{x}+i \vec{\sigma}, x_{i}^{\prime} \vec{\sigma}\right)=\left\{\left(\vec{x}-\vec{x}^{\prime}\right)^{2}+\left(z(\vec{x}+i \vec{\sigma}) \pm z\left(\vec{x}^{\prime}+i \vec{\sigma}\right)\right)^{2}\right\}^{3 / 2} \\
D_{0}\{z\}\left(\vec{x}+i \vec{\sigma}, \vec{x}^{\prime}+i \vec{\sigma}\right)=\left\{\left(\vec{x}-\vec{x}^{\prime}\right)^{2}+4 z^{2}(\vec{x}+i \vec{\sigma})\right\}^{3 / 2} .
\end{gathered}
$$

To estimate $V$, we separately consider the operators

$$
\begin{aligned}
& U_{j}^{(i)}\{\Omega, z\}(\vec{x}+i \vec{\sigma}) \\
& =\int \theta_{i}\left(\left|\vec{x}-\vec{x}^{\prime}\right|\right)\left\{\frac{x_{j}-x_{j}^{\prime}}{D_{+}\{z\}\left(\vec{x}+i \vec{\sigma}, \vec{x}^{\prime}+i \vec{\sigma}\right)}\right. \\
& \left.-\frac{x_{j}-x_{j}^{\prime}}{D_{+}\{z\}\left(\vec{x}+i \vec{\sigma}, \vec{x}^{\prime}+i \vec{\sigma}\right)}\right\} \Omega(\vec{x}+i \vec{\sigma}) d \vec{x}^{\prime}, \\
& W^{(i)}\{\Omega, z\}(x+i \vec{\sigma}) \\
& =\int \theta_{i}\left(\left|\vec{x}-\vec{x}^{\prime}\right|\right)\left\{\frac{z(\vec{x}+i \vec{\sigma})-z\left(\vec{x}^{\prime}+i \vec{\sigma}\right)}{D_{-}\{z\}\left(\vec{x}+i \vec{\sigma}, \vec{x}^{\prime}+i \vec{\sigma}\right)}\right. \\
& \left.-\frac{z(x+i \vec{\sigma})+z\left(\vec{x}^{\prime}+i \vec{\sigma}\right)}{D_{+}\{z\}\left(\vec{x}+i \vec{\sigma}, x^{\prime}+i \vec{\sigma}\right)}\right\} \Omega\left(\vec{x}^{\prime}+i \vec{\sigma}\right) d \vec{x}^{\prime},
\end{aligned}
$$

where the functions $\theta_{i}$ defined in (6.8) separate the contributions at short and large distances. Like in two dimensions, the two terms of $U_{j}^{(1)}(j=1,2)$ and of $W^{(1)}$ and $W^{(2)}$ are estimated separately. We have

$$
\begin{aligned}
& \left\|U_{j}^{(1)}\{\Omega, z\}-U_{j}^{(1)}\{\tilde{\Omega}, \tilde{z}\}\right\|_{s} \\
& \quad \leq C(\gamma)\left\{\left(\frac{\|z\|_{s}}{h}+\frac{\|\nabla \eta\|_{s}}{h}\right)\|\Omega-\tilde{\Omega}\|_{s}+\frac{\|\delta \eta\|_{s}}{h}\|\tilde{\Omega}\|_{s}+\|\tilde{\Omega}\|_{s}\|\nabla \delta \eta\|_{s}\right\}
\end{aligned}
$$


The first term of $W^{(i)}$, denoted by $W_{1}^{(i)}$, is estimated like in [10]:

$$
\begin{aligned}
\| W_{1}^{(i)}\{\Omega, z\}- & W_{1}^{(i)}\{\tilde{\Omega}, z\} \|_{s} \\
\leq C\left(R_{2}\right)\left\{\left(\frac{\|\delta \eta\|_{s}}{h}+\frac{\|z\|_{s}}{h}\right)\|\Omega-\tilde{\Omega}\|_{s}\right. & \\
& \left.\quad+\|\tilde{\Omega}\|_{s}\left(\frac{\|\nabla(\eta-\tilde{\eta})\|_{s}}{h}+\|\eta-\tilde{\eta}\|_{s} / h\right)\right\}
\end{aligned}
$$

In the second term of $W^{(i)}$, denoted by $W_{2}^{(i)}$, we use for $|\eta|_{s}<\gamma h$,

$$
D_{+} \mid \geq(1-\gamma)^{3} h^{3}
$$

in $W_{2}^{(1)}$ and

$$
\left|D_{+}\right| \geq\left(\left(\vec{x}-\vec{x}^{\prime}\right)^{2}-4 \gamma^{2} h^{2}\right)^{3 / 2}
$$

in $W_{2}^{(2)}$. We thus get

$$
\text { (6.66) } \begin{aligned}
\mid W_{2}^{(1)}\{\Omega, z\}- & \left.W_{2}^{(1)}\{\tilde{\Omega}, \tilde{z}\}\right|_{s} \\
& \leq C\left\{\frac{|z|_{s}}{h(1-\gamma)^{3}}|\delta \Omega|_{s}+\frac{|\delta \eta|_{s}}{h(1-\gamma)^{3}}|\Omega|_{s}+\frac{|\delta \eta|_{s}}{h(1-\gamma)^{7}}|\Omega|_{s}\right\}
\end{aligned}
$$

and

$$
\begin{aligned}
\left|W_{2}^{(2)}\{\Omega, z\}-W_{2}^{(2)}\{\tilde{\Omega}, \tilde{z}\}\right|_{s} \leq & C\left\{|\delta \Omega|_{s}|z|_{s}+|\Omega|_{s}|\delta \eta|_{s}\right\} \int_{\rho>2 h} \frac{\rho d \rho}{\left.\left(\rho^{2}-4 \gamma^{2} h^{2}\right)\right|^{3 / 2}} \\
& +C|\Omega|_{s}|\delta \eta|_{s} h^{2} \int_{\rho>2 h} \frac{\left(\rho^{2}+4 h^{2}\right) \rho d \rho}{\left(\rho^{2}-4 \gamma^{2} h^{2}\right)^{3 / 2}} \\
\leq & \frac{C}{\left(1-\gamma^{2}\right)^{1 / 2}}\left(|\delta \eta|_{s} \frac{|z|_{s}}{h}+|\Omega|_{s} \frac{|\delta \eta|_{s}}{h}\right) \\
& +\frac{C}{\left(1-\gamma^{2}\right)^{2}}|\Omega|_{s} \frac{|\delta \eta|_{s}}{h}
\end{aligned}
$$

To estimate the difference,

(6.68) $\Delta=\left(W_{2}^{(i)}\{\Omega, z\}-W_{2}^{(i)}\{\tilde{\Omega}, \tilde{z}\}\right)(\vec{x}+i \vec{\sigma})-\left(W_{2}^{(i)}\{\Omega, z\}-W_{2}^{(i)}\{\tilde{\Omega}, \tilde{z}\}\right)\left(\vec{x}^{\prime}+i \vec{\sigma}\right)$.

We notice that

$$
\int_{0}^{\infty} \frac{2 z(\vec{x}+i \vec{\sigma}) \Omega(\vec{x}+i \vec{\sigma})}{\left(\rho^{2}+4 z^{2}(\vec{x}+i \vec{\sigma})\right)^{3 / 2}} \rho d \rho=2 \pi \Omega(\vec{x}+i \vec{\sigma}) .
$$

We then rewrite the difference $\Delta$ like in (6.24):

$$
\Delta=\sum_{i=1}^{2} \Delta_{1}^{(i)}+\Delta_{2}+\sum_{i=1}^{2} \Delta_{3}^{(i)}+\Delta_{4}+\sum_{i=1}^{2} \Delta_{5}^{(i)}+\Delta_{6}
$$


where

$$
\begin{gathered}
\Delta_{1}^{(i)}=\int \theta_{i}(|\vec{x}-\vec{q}|)\left\{\frac{(z(\vec{x}+i \vec{\sigma})+z(\vec{q}+i \vec{\sigma})) \delta \Omega(\vec{q}+i \vec{\sigma})}{D_{+}\{z\}(\vec{x}+i \vec{\sigma}, \vec{q}+i \vec{\sigma})}\right. \\
\left.-\frac{2 z(\vec{x}+i \vec{\sigma}) \delta \eta(\vec{q}+i \vec{\sigma})}{D_{0}\{z\}(\vec{x}+i \vec{\sigma}, \vec{q}+i \vec{\sigma})}\right\} d \vec{q} \\
-\int \theta_{i}\left(\left|\vec{x}^{\prime}-\vec{q}\right|\right)\left\{\frac{\left(z\left(\vec{x}^{\prime}+i \sigma\right)+z(q+i \sigma)\right) \delta \Omega(\vec{q}+i \vec{\sigma})}{D_{+}\{z\}\left(x^{\prime}+i \sigma, q+i \sigma\right)}\right. \\
\left.-\frac{2 z\left(\vec{x}^{\prime}+i \vec{\sigma}\right) \delta \Omega(\vec{q}+i \vec{\sigma})}{D_{0}\{z\}\left(\vec{x}^{\prime}+\vec{\sigma}, \vec{q}+i \vec{\sigma}\right)}\right\} d \vec{q}
\end{gathered}
$$

$$
\begin{gathered}
\Delta_{2}=-\pi\left(\delta \Omega(\vec{x}+i \vec{\sigma})-\delta \Omega\left(\vec{x}^{\prime}+i \vec{\sigma}\right)\right) \\
\Delta_{3}^{(i)}=\int \theta_{i}(|x-q|)\left\{\frac{(\delta z(\vec{x}+i \vec{\sigma})+\delta z(\vec{q}+i \vec{\sigma})) \vec{\Omega}(\vec{q}+i \vec{\sigma})}{D_{+}\{z\}(\vec{x}+i \vec{\sigma}, \vec{q}+i \vec{\sigma})}\right. \\
\left.-\frac{2 \delta z(\vec{x}+i \vec{\sigma}) \tilde{\Omega}(\vec{x}+i \vec{\sigma})}{D_{0}\{z\}(\vec{x}+i \sigma, \vec{q}+i \vec{\sigma})}\right\} d \vec{q} \\
-\int \theta_{i}(|x-q|)\left\{\frac{\left(\delta z\left(\vec{x}^{\prime}+i \vec{\sigma}\right)+\delta z(\vec{q}+i \vec{\sigma})\right) \tilde{\Omega}(q+i \vec{\sigma})}{D_{+}\{z\}\left(\vec{x}^{\prime}+i \vec{\sigma}, \vec{q}+i \vec{\sigma}\right)}\right. \\
\left.-\frac{2 \delta z\left(\vec{x}^{\prime}+i \vec{\sigma}\right) \tilde{\Omega}(\vec{x}+i \vec{\sigma})}{D_{0}\{z\}\left(\vec{x}^{\prime}+i \vec{\sigma}, \vec{q}+i \vec{\sigma}\right)}\right\} d q
\end{gathered}
$$

$$
\begin{gathered}
\Delta_{4}=\left(\frac{\delta \eta(\vec{x}+i \vec{\sigma})}{z(\vec{x}+i \vec{\sigma})} \tilde{\Omega}(\vec{x}+i \vec{\sigma})-\frac{\delta \eta\left(\vec{x}^{\prime}+i \vec{\sigma}\right)}{z\left(\vec{x}^{\prime}+i \vec{\sigma}\right)} \tilde{\Omega}(x+i \sigma)\right) \\
\Delta_{5}^{(i)}=\int \theta_{i}(|\vec{x}-\vec{q}|)\left\{( \tilde { z } ( \vec { x } + i \vec { \sigma } ) + \vec { z } ( \vec { q } + i \vec { \sigma } ) ) \left(\frac{1}{D_{+}\{z\}(\vec{x}+i \vec{\sigma}, \vec{q}+i \vec{\sigma})}\right.\right. \\
\left.-\frac{1}{D_{+}\{\tilde{z}\}(\vec{x}+i \vec{\sigma}, \vec{q}+i \vec{\sigma})}\right) \tilde{\Omega}(\vec{q}+i \sigma) \\
-2 \tilde{z}(\vec{x}+i \vec{\sigma})\left(\frac{1}{D_{0}\{z\}(\vec{x}+i \vec{\sigma}, \vec{q}+i \vec{\sigma})}\right. \\
\left.\left.-\frac{1}{D_{0}\{\tilde{z}\}(\vec{x}+i \vec{\sigma}, \vec{q}+i \vec{\sigma})}\right) \tilde{\Omega}(x+i \sigma)\right\} d \vec{q} \\
-\int \theta_{i}\left(\left|\vec{x}^{\prime}-\vec{q}\right|\right)\left\{( z ( \vec { x } ^ { \prime } + i \vec { \sigma } ) + z ( \vec { q } + i \vec { \sigma } ) ) \left(\frac{1}{D_{+}\{z\}\left(\vec{x}^{\prime}+i \vec{\sigma}, \vec{q}+i \vec{\sigma}\right)}\right.\right. \\
-2 \tilde{z}\left(\vec{x}^{\prime}+i \vec{\sigma}\right)\left(\frac{1}{D_{+}\{\tilde{z}\}\left(\vec{x}^{\prime}+i \vec{\sigma}, \vec{q}+i \vec{\sigma}\right)}\right) \tilde{\Omega}(q+i \sigma) \\
-\frac{1}{D_{0}\{z\}\left(\vec{x}^{\prime}+i \vec{\sigma}, \vec{q}+i \vec{\sigma}\right)} \\
\left.\left.-\frac{1}{D_{0}\{\tilde{z}\}\left(\vec{x}^{\prime}+i \vec{\sigma}, \vec{q}+i \vec{\sigma}\right)}\right) \tilde{\Omega}\left(\vec{x}^{\prime}+i \vec{\sigma}\right)\right\} d \vec{q},
\end{gathered}
$$

$$
\Delta_{6}=\frac{\tilde{\Omega}(\vec{x}+i \vec{\sigma})}{z(\vec{x}+i \vec{\sigma})} \delta \eta(\vec{x}+i \vec{\sigma})-\frac{\tilde{\Omega}\left(x^{\prime}+i \sigma\right)}{z\left(\vec{x}^{\prime}+i \vec{\sigma}\right)} \delta \eta(\vec{x}+i \vec{\sigma})-\delta \eta(\vec{x}+i \vec{\sigma})
$$


We obtain

$$
\left.\left\|W_{2}\{\Omega, z\}-W_{2}\{\tilde{\Omega}, \tilde{z}\}\right\|_{s} \leq C\left(\gamma, R_{2}\right)\|\delta \eta\|_{s}+\|\Omega\|_{s}\|\delta \eta\|_{s} / h\right),
$$

where $C\left(\gamma, R_{2}\right)$ is a constant uniformly bounded for $\gamma \in\left[0, \gamma_{0}\right], \gamma_{0}<1$, and $R_{2}<R, R$ arbitrary.

To estimate $U_{j}^{(2)}$, we write

$$
\begin{array}{r}
U_{j}^{(2)}\{\Omega, z\}(\vec{x}+i \vec{\sigma})=\int \theta_{2}(|\vec{x}-\vec{x}|) \frac{\left(x_{j}-x_{j}^{\prime}\right)\left(D_{-}\{z\}-D_{+}\{z\}\right)\left(\vec{x}+i \vec{\sigma}, \vec{x}^{\prime}+i \vec{\sigma}\right)}{D_{+}\{z\}\left(\vec{x}+i \vec{\sigma}, \vec{x}^{\prime}+i \vec{\sigma}\right) D_{-}\{z\}\left(\vec{x}+i \sigma, \vec{x}^{\prime}+i \vec{\sigma}\right)} \\
\times \Omega\left(\vec{x}^{\prime}+i \vec{\sigma}\right) d \vec{x}^{\prime}
\end{array}
$$

and we use

(6.79)

$$
\frac{\left|D_{+}\{z\}-D_{-}\{z\}\right|}{\left|D_{+}\{z\} D_{-}\{z\}\right|} \leq h^{2} \frac{\left\{2\left|\vec{x}-\vec{x}^{\prime}\right|^{2}+2 h^{2}+C\left|x-x^{\prime}\right|\left(\left|\vec{x}-\vec{x}^{\prime}\right|^{2}+4 h^{2}\right)^{1 / 2}\right\}}{\left|x-x^{\prime}\right|^{3}\left(\left|x-x^{\prime}\right|^{2}-4 \gamma^{2} h^{2}\right)^{2}} .
$$

Thus

(6.80)

$$
\begin{aligned}
\left|U_{j}^{(2)}\{\delta \Omega, z\}\right| \leq c|\delta \Omega|_{s} h^{2}\left\{\int_{\rho>2 h} \frac{\rho d \rho}{\left(\rho^{2}-4 \gamma^{2} h^{2}\right)^{2}}\right. & +h^{2} \int_{\rho>2 h} \frac{\rho d \rho}{\rho^{2}\left(\rho^{2}-4 \gamma^{2} h^{2}\right)^{2}} \\
& \left.+h \int_{\rho>2 h} \frac{\rho d \rho}{\rho\left(\rho^{2}-4 \gamma^{2} h^{2}\right)^{2}}\right\} \\
& \leq|\delta \eta|_{s} \frac{1}{\left(1-\gamma^{2}\right)} .
\end{aligned}
$$

Similarly,

$$
\begin{aligned}
& \left|U_{j}^{(2)}\{\Omega, z\}-U_{j}^{(2)}\{\Omega, \tilde{z}\}\right|_{s} \\
& \quad \leq C\left(R_{2}\right)|\Omega|_{2}\left\{\frac{1}{1-\gamma^{2}} \frac{|\delta \eta|_{s}}{h}+\frac{1}{\left(1-\gamma^{2}\right)^{3 / 2}} \frac{|\delta \eta|_{s}}{h}+\frac{1}{\left(1-\gamma^{2}\right)^{2}} \frac{|\delta \eta|_{s}}{h}\right\}
\end{aligned}
$$

To estimate

$$
\left(U_{j}^{(2)}\{\Omega, z\}-U_{j}^{(2)}\{\tilde{\Omega}, \tilde{z}\}\right)(\vec{x}+i \vec{\sigma})-\left(U_{j}^{(2)}\{\Omega, z\}-U_{j}^{(2)}\{\tilde{\Omega}, \tilde{z}\}\right)\left(\vec{x}^{\prime}+i \vec{\sigma}\right),
$$

we rewrite $U_{j}^{(2)}\{\Omega, z\}(\vec{x}+i \vec{\sigma})$ in the form

$$
\begin{aligned}
& U_{j}^{(2)}\{\Omega, z\}(x+i \sigma) \\
& =\int \theta_{2}(|x-q|)\left\{\frac{\left(x_{j}-q_{j}\right)\left(D_{-}\{z\}-D_{+}\{z\}\right)(\vec{x}+i \vec{\sigma}, q+i \vec{\sigma})}{D_{+}\{z\}(\vec{x}+i \vec{\sigma}, \vec{q}+i \vec{\sigma}) D_{-}\{z\}(\vec{x}+i \vec{\sigma}, \vec{q}+i \vec{\sigma})}-\left(x_{j}-q_{j}\right) \Omega(\vec{x}+i \vec{\sigma})\right. \\
& \left.\quad \times \frac{\left\{|\vec{x}-\vec{q}|^{3}(1+(\vec{x}-\vec{q}) /|\vec{x}-\vec{q}| \cdot \nabla z(\vec{x}+i \vec{\sigma}))^{3 / 2}-D_{0}\{z\}(\vec{x}+i \vec{\sigma}, \vec{q}+i \vec{\sigma})\right\}}{D_{0}\{z\}(\vec{x}+i \vec{\sigma}, \vec{q}+i \vec{\sigma})|x-q|^{3}(1+(\vec{x}-\vec{q}) /|\vec{x}-\vec{q}| \cdot \nabla z(\vec{x}+i \vec{\sigma}))^{3 / 2}}\right\} d \vec{q},
\end{aligned}
$$

where the last integral vanishes by symmetry. After lengthy but straightforward calculations, we get

$$
\left\|U_{j}^{(2)}\{\Omega, z\}-U_{j}^{(2)}\{\tilde{\Omega}, \tilde{z}\}\right\|_{s} \leq c\left(\gamma, R_{2}\right)\left\{\|\Omega-\tilde{\Omega}\|_{s} \frac{\|\delta \eta\|_{s}}{h} \frac{\|\nabla \delta \eta\|_{s}}{h}\right\} .
$$

This completes the proof of (6.55). 
The kernel $\mathcal{G}$, defined in (3.43), and its gradient belong to $L^{1}\left(R^{2}+i \sigma\right)$. It is thus sufficient to estimate the operators $A_{j}\{z\} \xi$ for $j=1,2,3$. These estimates follow from those on $V\{\Omega, z\}$. Thus,

$$
\begin{gathered}
\left\|A_{j}\{z\} \xi-A_{j}\{z\} \tilde{\xi}\right\|_{s} \leq \alpha C\left(\gamma, R_{2}\right)\left(\gamma+\|\nabla \eta\|_{s}\right)\|\xi-\tilde{\xi}\|_{s} \\
\left\|A_{j}\{z\} \xi-A_{j}\{\tilde{z}\} \xi\right\|_{s} \leq \alpha C\left(\gamma, R_{2}\right)\|\xi\|_{s}\left(\frac{\|\eta-\tilde{\eta}\|_{s}}{h}+\frac{\|\nabla(\eta-\tilde{\eta})\|_{s}}{h}\right) .
\end{gathered}
$$

To estimate the second term of (3.42), we proceed as in $\S 5$ and get the same estimates (5.19)-(5.21) with the norm $\||\cdot|\|_{s}$ replaced by the Hölder norms $\|\cdot\|_{s}$. This leads to

THEOREM 6.2. There exists a constant $k_{0}$ such that for initial conditions $\left(z_{0}=\right.$ $\left.h+\eta+0, \nabla z_{0}, \Omega_{0}\right)$ whose analytic continuation belongs to $B_{s_{0}}^{(1)} \times\left(B_{s_{0}}^{(1)}\right)^{2} \times\left(B_{s_{0}}^{(1)}\right)^{3}$ with the conditions $\left|\operatorname{Im} \nabla z_{0}\right|_{s}<K<1,\left\|\eta_{0}\right\|_{s}<\gamma h, \gamma \leq \gamma_{0}<1$, and

$$
\alpha\left\{\gamma+\|\nabla \eta\|_{s}\right\}<k_{0}
$$

there exists a constant $a$, such that for $|t|<a\left(s_{0}-s\right)$, the system (3.42) has a unique solution $(z, \nabla z, \Omega)$ which is an analytic function of $t$ with values in $B_{s_{0}}^{(1)} \times$ $\left(B_{s_{0}}^{(1)}\right) \times\left(B_{s_{0}}^{(1)}\right)^{3}$.

REMARK. In the presence of a bottom, the initial interface perturbation is not required to vanish at infinity as it is the case when the Rayleigh-Taylor (or the Kelvin-Helmholtz) problem is considered in the entire space. The reason is the faster decay of the gradient of the Green function of the Poisson equation which relates a divergence free velocity and vorticity. This insures integral-convergence without additional assumptions. A similar situation is encountered in the proof of existence in the large of a unique solution to the two-dimensional Euler equation with smooth initial data (Hölder continuous vorticity) (Wolibner [16], Kato [17]). In the entire space, the initial vorticity is required to be bounded in $L^{1}\left(R^{2}\right)$ (Bardos and Benachour [18]). This condition is relaxed when the flow is considered in half a plane or a strip (Sulem [19]).

7. Open problems. The existence theorems presented in $\S \S 5$ and 6 assume an initial interface with sufficiently small corrugations. One can, however, notice in two dimensions that, defining $\mu(x, t)$ as a primitive of $\Omega(x, t),(2.19)$ (b) may be written in the form

$$
\frac{\partial \mu}{\partial t}(x, t)+\alpha \int \frac{\partial G}{\partial x^{\prime}}\left(x, x^{\prime}\right) \frac{\partial \mu}{\partial t}\left(x^{\prime}, t\right) d x^{\prime}=F(x, t) .
$$

When the problem is considered in the real domain, the operator

$$
\mathcal{A} \frac{\partial \mu}{\partial t}(x)=\int \frac{\partial G}{\partial x^{\prime}}\left(x, x^{\prime}\right) \frac{\partial \mu}{\partial t}\left(x^{\prime}\right) d x^{\prime}
$$

has all its eigenvalues with a modulus strictly larger than one. This property is a consequence of the irrotationality of the flow in the two domains separated by the interface $[20,21]$. For $\alpha \leq 1$, the operator $1+\alpha \mathcal{A}$ is thus invertible. However, the property is not necessarily preserved when the equations are continued to the complex domain if the norm of $\alpha A$ is not smaller than 1 . 
Another questions concerns the shallow water limit [22] for the Rayleigh-Taylor problem in the presence of a horizontal bottom. It corresponds to the situation where $t=\varepsilon^{-1 / 2} \tilde{t}, y(x, t)=\varepsilon y^{(\varepsilon)}(x, t)$ and $\Omega(x, t)=\varepsilon^{1 / 2} \Omega^{(\varepsilon)}(x, t)$ with $\varepsilon \rightarrow 0$. One can show that if $y^{(\varepsilon)}$ and $\Omega^{(\varepsilon)}$ tend uniformly to $\eta^{(0)}$ and $\Omega^{(0)}$, then $\tilde{V}_{1}=\varepsilon^{-1 / 2} V_{1} \rightarrow$ $\Omega^{(0)}$ and $\tilde{V}_{2}=\varepsilon^{-3 / 2} V_{2} \rightarrow \partial\left(\Omega^{(0)} y^{(0)}\right) / \partial x+y^{(0)} \partial \Omega^{(0)} / \partial x$. The equations of motion then reduce to

$$
\frac{\partial y^{(0)}}{\partial t}+\frac{\partial}{\partial x}\left(\Omega^{(0)} y^{(0)}\right)=0, \quad \frac{\partial \Omega^{(0)}}{\partial t}+\frac{\partial}{\partial x}\left(\frac{\Omega^{(0) 2}}{2}+\frac{2 \alpha}{\alpha+1} g y^{(0)}\right)=0
$$

where, in the shallow water limit, $\Omega^{(0)}$ identifies with the rescaled horizontal component of the velocity jump $\varepsilon^{-1 / 2}[u]$ across the interface.

Similarly, in three dimensions, if $z^{(\varepsilon)}(x, y, t)=\varepsilon z(x, y, t)$ and $\Omega^{(\varepsilon)}(x, y, t)=$ $\varepsilon^{1 / 2} \Omega(x, y, t)$ have uniform limits $z^{(0)}$ and $\Omega^{(0)}$ when $\varepsilon \rightarrow 0$, then $\tilde{V}_{1}=\varepsilon^{1 / 2} V_{1} \rightarrow$ $-\Omega^{(0)}, \tilde{V}_{2}=-\varepsilon^{1 / 2} V_{2} \rightarrow \Omega_{1}^{(0)}$ and

$$
\tilde{V}_{3}=\varepsilon^{-3 / 2} V_{3} \rightarrow \frac{1}{2}\left\{\frac{\partial}{\partial x}\left(z^{(0)} \Omega_{2}^{(0)}\right)+z^{(0)} \frac{\partial \Omega_{2}^{(0)}}{\partial x}+\frac{\partial}{\partial y}\left(z^{(0)} \Omega_{1}^{(0)}\right)+z^{(0)} \frac{\partial \Omega_{1}^{(0)}}{\partial y}\right\} \text {. }
$$

Noticing that in the shallow water limit $\Omega_{1}^{(0)}=\left[u_{2}^{(0)}\right]$ and $\Omega_{2}^{(0)}=-\left[u_{1}^{(0)}\right]$ with $u_{1}^{(0)}=\lim _{\varepsilon \rightarrow 0} \varepsilon^{-1 / 2} u_{1}, u_{2}^{(0)}=\lim _{\varepsilon \rightarrow 0} \varepsilon^{-1 / 2} u_{2}$, and taking into account that the flow is irrotational in the two subdomains, we recover the Saint-Venant equations:

$$
\begin{aligned}
& \frac{\partial z^{(0)}}{\partial t}+\nabla \cdot\left(U^{(0)} z^{(0)}\right)=0, \\
& \frac{\partial}{\partial t} U^{(0)}+U^{(0)} \cdot \nabla U^{(0)}+\frac{2 \alpha}{\alpha+1} g \nabla z^{(0)}=0 \\
& \quad \text { with } U^{(0)}=\left(\left[u_{1}^{(0)}\right],\left[u_{2}^{(0)}\right]\right) .
\end{aligned}
$$

However, we did not succeed in proving uniform convergence of the solution of the Rayleigh-Taylor problem when $\varepsilon \rightarrow 0$. Indeed, estimates (6.4), (6.5) and (6.55) do not lead to uniform estimates for $\tilde{V}_{2}=\varepsilon^{-3 / 2} V_{2}$ in two dimensions or $\tilde{V}_{3}=\varepsilon^{-3 / 2} V_{3}$ in three dimensions in terms of the Hölder norm of $\Omega^{(\varepsilon)}=\varepsilon^{-1 / 2} \Omega$. Uniform estimates have only been obtained in terms of the gradient of $\Omega$. The CauchyKowalewski Theorem concerning first order systems $\nabla \Omega$ must be considered as an additional unknown. But the equation for $\nabla \Omega$ introduces higher order derivatives such as $\nabla \nabla z$ (or $y_{x x}$ in two dimensions) as additional variables inside a first order operator and the hierarchy can probably not be closed. Clearly, the difficulty originates from the necessity of considering the equation for the interface gradient $\left(y_{x}\right.$ or $\left.\nabla z\right)$ to prove well-posedness of the Rayleigh-Taylor problem.

These two difficulties disappear for $\alpha=1$ (water waves problem) in two dimensions when the approach of Ovsjannikov [3] and Kano and Nishida [4] is used. This method is based on a conformal mapping of the flow domain on a strip. It is, however, unclear how to extend this method, even in two dimensions to the Rayleigh-Taylor problem where the coupled dynamics of the two fluids must be considered. 
Appendix. Large distance behaviour of the Green function of the equation:

$$
f(\vec{x})+\alpha \int_{R^{2}} \frac{2 h}{\left(\left|\vec{x}-\vec{x}^{\prime}\right|^{2}+4 h^{2}\right)^{3 / 2}} f\left(\vec{x}^{\prime}\right) d \vec{x}^{\prime}=S(\vec{x}) .
$$

The solution is obtained by taking the two-dimensional Fourier transform (F.T.) of (A.1) and noticing that the F.T. of the kernel is equal to $e^{-2 h k}$. One obtains

$$
f(\vec{x})=\int \mathcal{G}\left(\vec{x}^{\prime}\right) S\left(\vec{x}-\vec{x}^{\prime}\right) d \vec{x}^{\prime}
$$

with $(x=|\vec{x}|)$

$$
\mathcal{G}(\vec{x})=\int_{R^{2}} \frac{e^{-i \vec{k} \cdot \vec{x}}}{1+\alpha e^{-2 k h}} d \vec{k}=\int_{0}^{\infty} \frac{k J_{0}(k x)}{1+\alpha e^{-2 k h}} d k .
$$

The asymptotic behaviour of $\mathcal{G}(x)$ when $x \rightarrow \infty$ is obtained by using iteratively the following procedure:

(i) Make the substitution

$$
J_{0}(k x)=\frac{1}{x} \frac{d}{d k}\left(k J_{1}(x k)\right) .
$$

(ii) Integrate by parts.

(iii) Make the substitution

$$
J_{1}(k x)=-\frac{1}{x} \frac{d}{d k} J_{0}(k x) .
$$

Defining

$$
f(k)=\frac{2 h}{1+\alpha e^{-2 h k}}
$$

and

$$
g(k)=\frac{d f}{d k}=\frac{4 \alpha h^{2} e^{-2 h k}}{\left(1+\alpha e^{-2 h k}\right)^{2}}
$$

we have

$$
\begin{aligned}
\int_{0}^{\infty} f(k) k J_{0}(k x) d k & =\frac{1}{x} \int_{0}^{\infty} f(k) \frac{d}{d k}\left(k J_{1}(k x)\right) d k \\
& =\frac{1}{x} \int_{0}^{\infty} g(k) k J_{1}(k x) d k \\
& =\frac{1}{x^{2}} \int_{0}^{\infty} k g(k) \frac{d}{d k} J_{0}(k x) d k \\
& =-\frac{1}{x^{2}} \int_{0}^{\infty} \frac{d}{d k}(k g(k)) J_{0}(k x) d k \\
& =-\frac{1}{x^{3}} \int_{0}^{\infty} \frac{1}{k} \frac{d}{d k}(k g(k)) \frac{d}{d k}\left(k J_{1}(k x)\right) d k \\
& =\frac{1}{x^{3}} \int_{0}^{\infty} \frac{d}{d k}\left(\frac{1}{k} \frac{d}{d k}(k g)(k)\right) k J_{1}(k x) d k \\
& =\frac{1}{x^{3}} \int_{0}^{\infty} F\left(\frac{\lambda}{x}\right) J_{1}(\lambda) \frac{d \lambda}{\lambda}
\end{aligned}
$$


with

$$
F\left(\frac{\lambda}{x}\right)=\lambda^{2} \frac{d}{d \lambda}\left\{\frac{1}{\lambda} \frac{d}{d \lambda}\left(\lambda g\left(\frac{\lambda}{x}\right)\right)\right\}
$$

and

$$
g(\lambda / x)=\alpha h e^{-2 h(\lambda / x)} /\left(1+\alpha e^{-2 h(\lambda / x)^{2}}\right) .
$$

$F(\lambda / x)$ is uniformly bounded in $x$ and $\lambda ; J_{1}(\lambda) \sim \lambda$ when $\lambda \rightarrow 0$ and $J_{1}(\lambda) \sim \lambda^{-1 / 2}$ when $\lambda \rightarrow \infty$. It follows that the integral in the last term of (A.9) is bounded. As a consequence, $\mathcal{G}(x) \in L^{1}\left(R^{2}\right)$.

\section{REFERENCES}

1. S. Chandrasekhar, Hydrodymamic and hydromagnetic stability, Clarendon Press, Oxford, 1961.

2. G. Birkhoff, Helmholtz and Taylor instability, Hydrodynamic Instability, Proc. Sympos. Appl. Math., vol. 13, Amer. Math. Soc., Providence, R.I., 1962, pp. 55-76.

3. L. V. Ovsjanikov, Arch. Mech. (Arch. Mech. Stos.) 26 (1974), 407-422.

4. T. Kano and T. Nishida, J. Math. Tokyo Univ. 19 (1979), 335-370.

5. V. I. Nalimov, Dokl. Akad. Nauk. SSSR 189 (1969), 45-48.

6. J. C. W. Rogers, Water waves; analytic solutions, uniqueness and continuous dependence on the data, Naval Ordinance Laboratory NSWC/WOL/TR 75-43, 1975.

7 V. I. Nalimov, Dinamika Splosh. Sredy 18 (1974), 104-210.

8. H. Yosihara, Publ. Res. Inst. Math. Sci. 18 (1982), 49-96.

9. Bui An Ton, Nonlinear Anal. Theor. Meth. Appl. 6 (1982), 335-347.

10. I. I. Bakenko and V. U. Petrovitch, Soviet Phys. Dokl. 24 (1969), 161-163.

11. L. V. Ovsjanikov, Dokl. Akad. Nauk. SSSR 200 (1971); Soviet Math. Dokl. 12 (1971), 1497-1502.

12. C. Sulem, P. L. Sulem, C. Bardos and U. Frisch, Comm. Math. Phys. 80 (1981), 485-516.

13. T. Nishida, J. Differential Geom. 12 (1977), 629-633.

14. M. S. Baouendi and C. Goulaouic, Comm. Partial Differential Equations 2 (1977), 11511162 .

15. L. Nirenberg, J. Differential Geom. 6 (1972), 561-576.

16. W. Wolibner, Math. Z. 37 (1933), 698-726.

17. T. Kato, Arch. Rational Mech. Anal. 25 (1967), 188-200.

18. C. Bardos and S. Benachour, Ann. Scuola Norm. Sup. Pisa Cl. Sci. (4) 4 (1977), 647-687.

19. C. Sulem, C. R. Acad. Sci. Paris Ser. A 287 (1978), 623-628.

20. M. Shiffer, Pacific J. Math. 7 (1957), 1187-1225; 9 (1959), 211-269.

21. G. R. Baker, D. I. Meiron and S. A. Orszag, J. Fluid Mech. 123 (1982), 477-501.

22. G. B. Whitham, Linear and nonlinear waves, Interscience, New York, 1973, p. 114.

Department of Mathematics, Ben Gurion University of the Negev, Beer SHEVA, ISRAEL (Current address of C. Sulem)

CNRS, Département de Mathématiques, Université de Nice, 06-Nice, France

School of Mathematical Sciences, Tel Aviv University, Ramat Aviv, Israel (Current address of P. L. Sulem)

CNRS, OBSERVATOIRE DE NiCE, 06-NicE, FranCE 